Revue des patrimoines

Le patrimoine religieux des $\mathrm{XIX}^{\mathrm{e}}$ et $\mathrm{XX} \mathrm{X}^{\mathrm{e}}$ siècles

\title{
À propos des reliquaires, questions de méthode
}

\section{Yves Gagneux}

\section{OpenEdition}

Journals

\section{Édition électronique}

URL : http://journals.openedition.org/insitu/9909

DOI : $10.4000 /$ insitu.9909

ISSN : 1630-7305

\section{Éditeur}

Ministère de la culture

\section{Référence électronique}

Yves Gagneux, "À propos des reliquaires, questions de méthode », In Situ [En ligne], 11 | 2009, mis en ligne le 22 octobre 2012, consulté le 23 avril 2019. URL : http://journals.openedition.org/insitu/9909 ; DOl : 10.4000/insitu.9909

Ce document a été généré automatiquement le 23 avril 2019.

\section{(c) (i) $\odot$}

In Situ Revues des patrimoines est mis à disposition selon les termes de la licence Creative Commons Attribution - Pas d'Utilisation Commerciale - Pas de Modification 4.0 International. 


\title{
À propos des reliquaires, questions de méthode
}

\author{
Yves Gagneux
}

1 Le culte des reliques est, dans l'imaginaire public, associé au Moyen Âge, à de beaux récits aimablement naïfs et à de remarquables pièces d'orfèvrerie conservées dans les musées et certains trésors d'églises ${ }^{1}$. Si la production religieuse des XIXe et XXe siècles a laissé peu de chefs-d'œuvre en France et suscité plus de sarcasmes que de légendes ou de récits poétiques, le culte voué aux reliques des saints n'a pour autant pas disparu après la Révolution et connaît au contraire, dans la seconde moitié du XIX ${ }^{e}$ siècle, un renouveau dont témoigne la présence de nombreux reliquaires. Les reliques des saints canonisés après 1800 , celles des saints martyrs des catacombes dont la diffusion connait alors une vigueur sans précédent, suppléent très largement aux destructions opérées par les révolutionnaires. Dans les églises progressivement rendues au culte après 1796, le clergé s'emploie à présenter ces vestiges avec une grande liberté, les destructions ayant beaucoup allégé le poids des traditions ou des choix antérieurs.

Comment inventorier ces reliquaires des deux derniers siècles qui abondent dans les églises françaises? Avant de s'interroger sur la manière de procéder, il convient de s'entendre sur les objectifs que l'on cherche à atteindre.

La notion d'inventaire recouvre en effet des acceptions distinctes. L'inventaire patrimonial désigne le recensement des biens publics à des fins de gestion: c'est par exemple la vocation initiale de la Conservation des œuvres d'art religieuses et civiles de la Ville de Paris (COARC) ${ }^{2}$ qui a été fondée en 1996 pour répondre à une observation de la cour des Comptes s'étonnant de l'ignorance où se trouvait cette commune de son patrimoine religieux. Le relevé est destiné d'une part à compléter les inventaires établis après la loi de séparation de l'Église et de l'État promulguée en 1905, de l'autre à faciliter la gestion de ces biens. Il n'est donc aucune justification scientifique à la création de ce service et le recensement pourrait se limiter à quelques indications permettant de localiser l'objet, d'en donner une estimation et de le conserver dans de bonnes conditions ${ }^{3}$; une photographie facilite l'identification en cas de vol, en particulier si elle montre un 
détail caractéristique, par exemple une altération, notamment pour les objets fabriqués en série.

Dans cette logique exclusivement gestionnaire, le relevé du détail des représentations, du style, et $a$ fortiori leur analyse sont inutiles ${ }^{4}$, ainsi que les informations historiques (date ou lieu d'exécution), sauf en cas d'incidence sur la valeur vénale des œuvres. En revanche, dimensions, matière, état de conservation auront plus d'importance. Ce relevé devrait être suivi d'une évaluation financière, à l'instar de celles des inventaires effectués en 1906, afin de fixer les priorités de restauration ou de protection des biens ${ }^{5}$. Dans cette optique, des éléments sans valeur vénale devraient être négligés et il serait par exemple injustifié de photographier ou d'enregistrer ces innombrables cartes en papier sur lesquelles est fixé le pétale d'une fleur laissée sur le reliquaire de sainte Thérèse.

Les objectifs d'un inventaire scientifique offrent a priori peu de points communs avec ceux de l'inventaire patrimonial. Ce dernier s'accompagne néanmoins presque toujours d'un travail fondamental de recherche, du fait de l'indispensable qualification du personnel qui le réalise. De plus, l'étude confère parfois à des œuvres qu'elle exhume de l'oubli une valeur financière. Le relevé des œuvres conservées dans les églises, surtout s'il s'accompagne d'une campagne photographique, offre aux chercheurs un outil sans équivalent. L'inventaire du patrimoine au sens où l'entend la Cour des Comptes n'est certes pas scientifique mais rien n'interdit de doubler ce travail de recherches susceptibles d'intéresser historiens, anthropologues ou historiens de l'art, si ce n'est le renchérissement des campagnes photographiques, car les objets les plus humbles présentent autant d'attrait que les châsses dorées pour celui qui s'intéresse aux manifestations du culte.

\section{La situation parisienne}

6 Nous traiterons ici d'un corpus de reliquaires établi en vue d'une recherche scientifique ${ }^{6}$, l'objectif patrimonial étant donc mis de côté. Par reliquaire, on entend généralement ces boîtes de forme variable, en bois ou en métal, qui renferment la relique et en autorisent le culte, définition dont on verra plus loin les limites. Ces objets appartiennent à différents propriétaires, et leur répartition répond à la complexité de l'histoire religieuse en France au cours des deux derniers siècles. De façon schématique, on pourrait dire que la Ville de Paris détient presque tous les objets des églises paroissiales antérieurs à 1906, qu'une partie notable du mobilier conservé dans la cathédrale Notre-Dame et dans quelques chapelles hospitalières relève de l'État, que les associations diocésaines gèrent la plupart des biens des églises construites au $\mathrm{XX}^{\mathrm{e}}$ siècle, que les nombreux ordres religieux implantés à Paris possèdent leur propres collections, qu'enfin quelques objets appartiennent aux desservants.

Durant la période de vandalisme liturgique des années 1960-1970, ces objets ont plutôt moins souffert que la statuaire, l'ameublement ou la lustrerie, car leurs dimensions restreintes permettaient de les remiser dans un placard ou des combles où l'oubli a assuré leur sauvegarde. Les destructions importantes ont seulement frappé quelques catégories bien précises d'objets liés au culte des reliques, comme les effigies en cire associées aux ossements des saints des catacombes, très à la mode au XIX ${ }^{\mathrm{e}}$ siècle mais fragiles, et en grande partie disparues - il n'en subsiste dans les églises parisiennes qu'une seule, dans un très mauvais état de conservation (fig. $\left.\mathbf{n}^{\circ} \mathbf{1}\right)\left(\right.$ fig. $\left.\mathbf{n}^{\circ} \mathbf{2}\right)$. Ce que l'on trouve aujourd'hui n'est pas représentatif de la production des deux siècles précédents. Paris ne donne pas 
davantage une image de la situation en France : les réalisations n'y sont presque jamais artisanales en raison de l'étroit contrôle exercé sur le culte des reliques; il n'existe presque aucun reliquaire de l'Ancien Régime et très peu postérieurs à la seconde guerre mondiale, la plupart datent du XIX ou du début du XX $\mathrm{XX}^{\mathrm{e}}$ siècle; les mutations de nombreux quartiers sont enfin d'une ampleur et d'une rapidité sans équivalent en province.

\section{Figure 1}

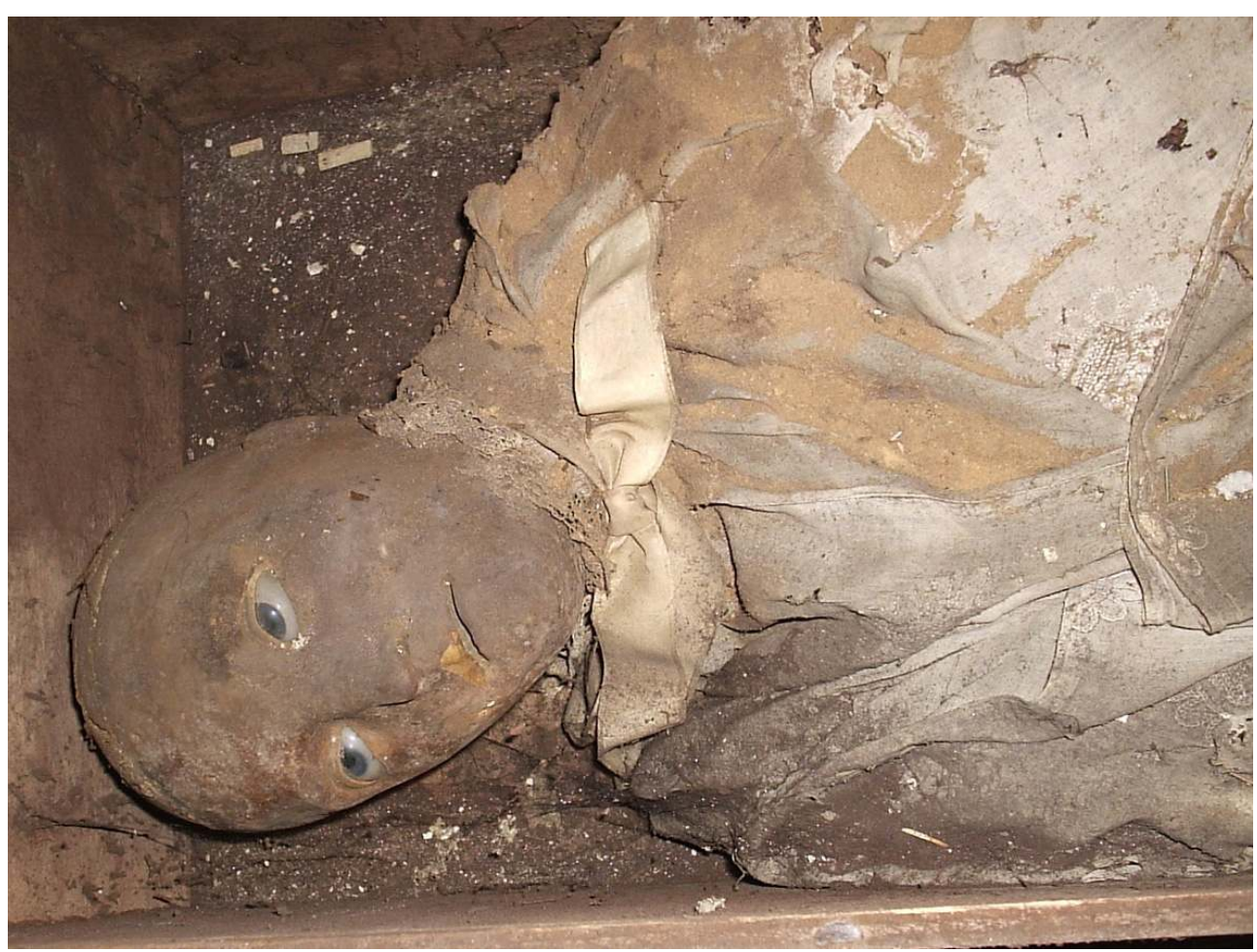

Détail du corps en cire de sainte Théodore. Paris, église Saint-Paul-Saint-Louis. Cette figurine très endommagée est aujourd'hui le seul qui subsiste à Paris des nombreux corps en cire figurant des martyrs des catacombes.

Phot. Y. Gagneux. (C) Y. Gagneux. 
Figure 2

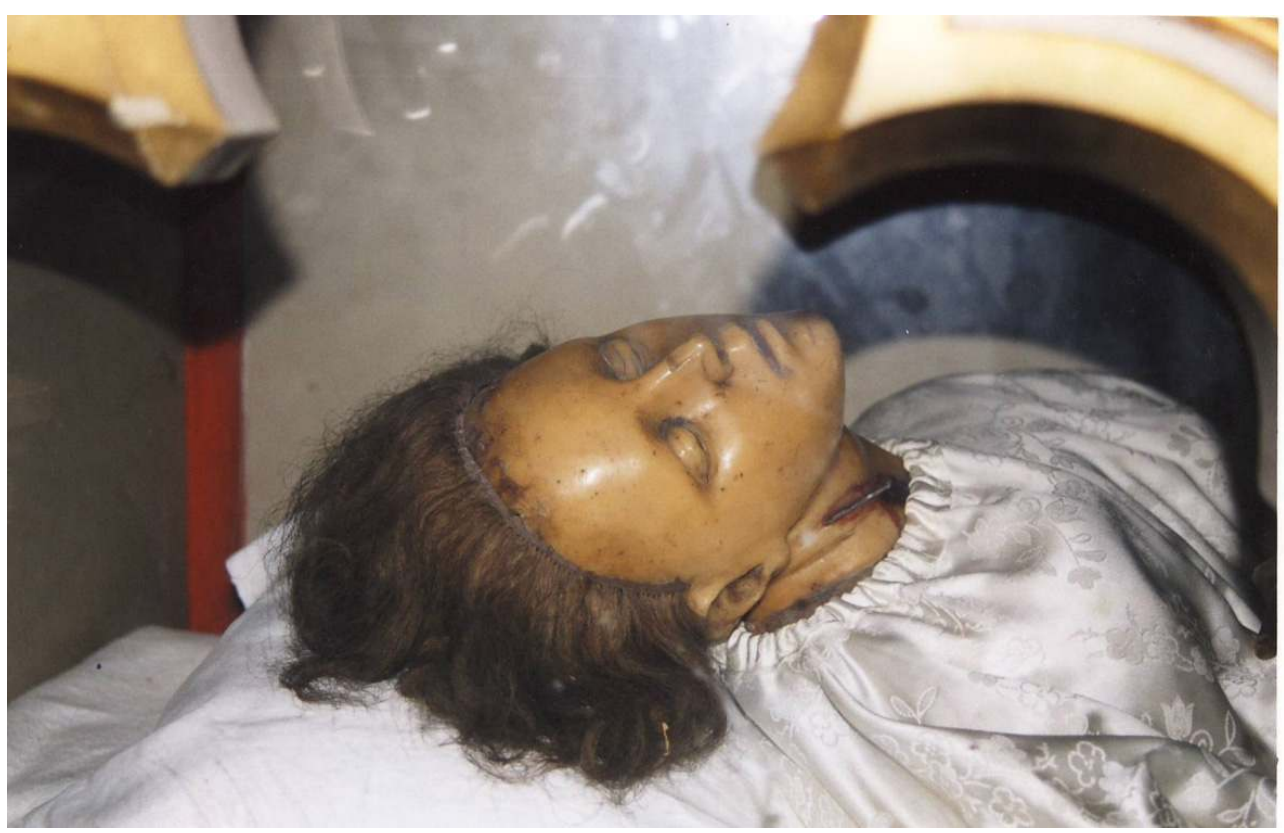

Détail du corps en cire de saint Clément, Oloron-Sainte-Marie (64). Voici, dans une église du SudOuest, un autre de ces corps dans un meilleur état de conservation.

Phot. Y. Gagneux. (c) Y. Gagneux.

Paris intègre aujourd'hui d'anciennes paroisses ouvrières ou rurales, la diversité du patrimoine religieux n'est donc pas pour surprendre. On ne peut par exemple qu'être frappé par les variations dans les dimensions, et l'on trouve ici des médaillons d'un ou deux centimètres de diamètre, là de gros reliquaires en bronze, ailleurs deux chapelles contiguës réunies pour le culte ${ }^{7}$. On observe de semblables écarts dans les formes, et tout éloigne la boîte ronde en métal blanc de l'effigie en cire placée sous un autel ou de l'ample gamme des structures métalliques proposée par les fabricants d'art religieux des XIXe et $\mathrm{XX}^{\mathrm{e}}$ siècles (fig. $\left.\mathbf{n}^{\circ} \mathbf{3}\right)\left(\right.$ fig. $\left.\mathbf{n}^{\circ} \mathbf{4}\right)$.

\section{Figure 3}

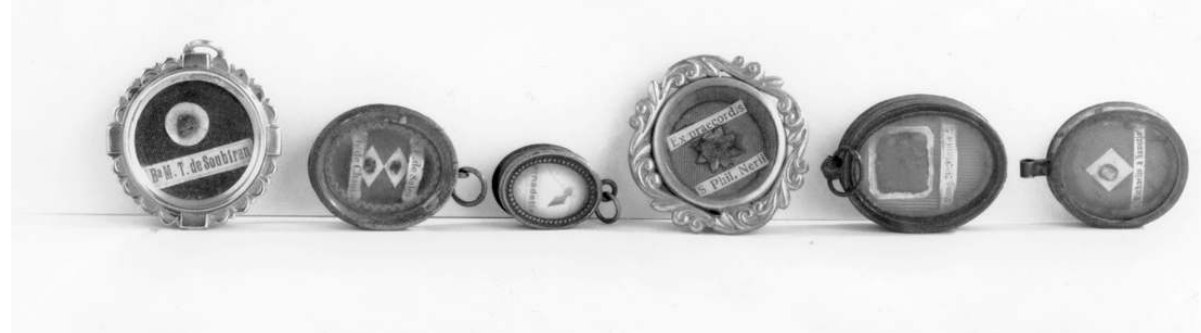

Exemple de très petits reliquaires. Paris, église Saint-Laurent. Celui de droite mesure moins d'un centimètre dans sa largeur. Ces objets sont destinés au transport des reliques mais peuvent être accrochés dans un reliquaire plus important, à côté d'autres médaillons, pour faire l'objet d'un culte. Les prescriptions ecclésiastiques imposent en principe une reconnaissance par l'ordinaire du lieu avant la présentation aux fidèles mais cette clause semble rarement observée si on en juge aux sceaux fermant ces médaillons.

Phot. Y. Gagneux. ( ) Y. Gagneux. 
Figure 4

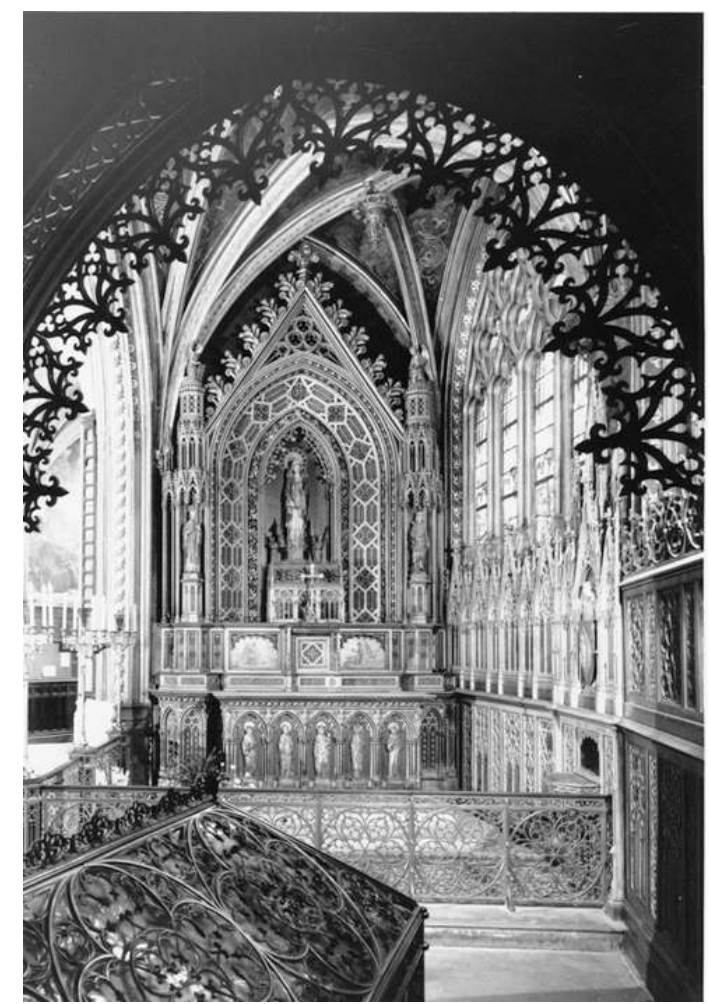

Chapelle de Sainte-Geneviève, Paris, église Saint-Étienne-du-Mont. Contrastant par son importance avec les médaillons, cet ensemble voué au culte du tombeau de sainte Geneviève occupe deux chapelles du déambulatoire de l'église Saint-Étienne-du-Mont.

Phot. Y. Gagneux. (c) Y. Gagneux.

\section{Quelles définitions?}

Les définitions évoluent et il n'est pas inutile de proposer un sens précis aux mots relique et reliquaire.

Dans la pensée catholique, le saint est habité par l'Esprit et susceptible d'intercéder auprès de Dieu en faveur des hommes. «Les fidèles doivent aussi vénérer les saints corps des martyrs et des autres saints qui vivent avec le Christ ; ils ont été des membres vivants du Christ et le temple du Saint Esprit [1Co 3,16;6,19;2Co 6,16] et seront ressuscités et glorifiés par lui pour la vie éternelle. Par eux Dieu accorde de nombreux biens aux hommes $^{8}$.»

11 Entre les premiers siècles du Christianisme et la fin du Moyen Âge, la notion de sainteté évolue fortement et la juridiction canonique n'est réellement fixée que par le concile de Trente (1545-1563), sous une forme qui reste opérante aujourd'hui. La canonisation tient lieu d'aboutissement à un parcours complexe et strictement déterminé. Si quelque personne meurt en odeur de sainteté, l'initiative de faire instruire un dossier revient à Rome exclusivement et, lorsque les conclusions sont satisfaisantes, la porte s'ouvre pour un procès de canonisation. La première étape sera la reconnaissance de l'héroïcité des vertus, condition indispensable pour que le pape décide de la béatification. Dans un 
second temps, et sous certaines conditions, le bienheureux sera proclamé saint. Le culte donc toute diffusion de relique - reste formellement proscrit avant la béatification.

Les vestiges d'un bienheureux ou d'un saint sont hiérarchisés par l'Église en quatre catégories, la plus importante étant celle des "reliques insignes ", à savoir le corps, la tête, le bras, l'avant-bras, le cœur, la langue, la main ou la jambe (mais pas le tibia ${ }^{9}$ ) ou la partie du corps dans laquelle le saint a subi le martyre, pourvu qu'elle soit entière et ne soit pas petite ${ }^{10}$. Le reste du corps forme les reliques corporelles non insignes. On appelle « reliques réelles» les objets à l'usage des saints durant leur vie terrestre, comme leurs vêtements, les objets sacrés ou profanes dont ils se servaient et surtout, les instruments de leur captivité ou de leur supplice. Les « reliques représentatives » sont le tombeau du saint et les linges, étoffes ou objets pieux mis au contact de leurs ossements, dans leur sépulcre, ou simplement des lampes de leur sanctuaire. L'Église établit ainsi une différence entre les restes humains et les objets ouvragés, sans lui accorder un caractère fondamental. Cette définition accorde une large place aux procédures ecclésiastiques et n'est pas dépourvue d'ambiguïtés, la question de l'authenticité pose notamment de réelles difficultés ${ }^{11}$.

La relique est à la fois le bien de l'Église, qui en dispose à son gré, mais aussi le saint en personne, réellement présent. Le récit des reconnaissances épiscopales souligne les précautions dans le maniement des reliques, traitées comme le serait le saint vivant. C'est dire s'il convient d'user d'égards lors d'une campagne d'inventaire et ce, quelle que soit l'attitude du clergé, parfois peu à l'aise devant des formes de culte devenues largement marginales.

Il faut noter que la propriété du reliquaire n'implique pas celle de la relique. Dans les églises paroissiales, les châsses en bronze ou laiton doré appartiennent aux communes depuis la dévolution des biens des fabriques, ces organismes créés lors du Concordat pour gérer les biens des paroisses, mais le transfert de propriété n'a pas concerné les reliques les inventaires parisiens menés à la suite de la loi de Séparation de l'Église et de l'État ne font d'ailleurs jamais mention du contenu ${ }^{12}$.

Les dictionnaires définissent le "reliquaire " ou la "châsse » comme la boîte où sont placées les reliques, sans plus de détail, et mentionnent le «buste-reliquaire » ou le "chef-reliquaire $~^{13}$. Le vocabulaire scientifique est plus large et bien plus précis mais souvent peu adapté à la situation des deux derniers siècles. Les dénominations (reliquaire anthropomorphe, médaillon reliquaire, reliquaire-monstrance, coffre-reliquaire, collierreliquaire, bague-reliquaire, croix-reliquaire, encolpion, staurothèque, ampoulereliquaire, lanterne-reliquaire, statue-reliquaire...) ont pour la plupart été conçues à partir d'objets médiévaux regroupés en lots cohérents selon des critères de représentation, de fonction, ou de forme. La confrontation de ces catégories au matériel des deux derniers siècles souligne leur empirisme. Ainsi, la notion de chef-reliquaire devient au XIXe siècle difficilement utilisable : elle s'applique parfaitement à des pièces d'orfèvrerie proches de celles des $14^{\text {ème }}$ ou $15^{\text {ème }}$ mais qui sont précisément des pastiches. Une création plus originale comme la tête en cire du curé d'Ars conservée à Notre-Damede-la-Salette représente une tête grandeur nature, elle se distingue néanmoins des productions des siècles antérieurs par le matériau (de la cire), la nature de la relique (ce n'est pas un fragment du crâne), la méthode de fabrication (un moulage opéré sur le visage), la présentation (sous une cloche de verre), etc. contexte et qui, lorsqu'ils servent encore au culte, sont au mieux intégrés à un système 
cultuel du XIXe siècle, rarement considéré par les spécialistes de l'orfèvrerie médiévale : il n'est pas possible d'appréhender le culte des reliques du Moyen Âge à travers son organisation matérielle. En revanche, l'exercice est envisageable pour les deux derniers siècles car non seulement subsiste le reliquaire (par exemple la boîte en bronze néogothique renfermant les os d'un saint), mais aussi tout le matériel associé à la dévotion aux restes saints, que les typologies traditionnelles n'associent pas aux reliques: les authentiques (les documents attestant la provenance des reliques), les sceaux, les peintures murales ornant la chapelle du saint, les statues ou vitraux liés au culte, les présentoirs à cierges, les brancards pour la procession, etc. On en vient à s'interroger sur le sens même de l'inventaire des reliquaires: est-il seulement destiné à l'étude des orfèvres et des bronziers, l'intérêt cultuel s'arrête-t-il au relevé du nom des saints, ou faut-il s'intéresser à l'expression du culte dans toute sa diversité ?

Il est d'ailleurs bien difficile de trouver un dénominateur commun au culte des reliques. Quel rapport entre une statuette d'ange tenant un tube en cristal, les boules des clochers où sont placées quelques fragments des saints patrons, ou les plaques d'autel ${ }^{14}$ composées d'un amalgame incorporant des fragments d'ossements? De surcroit, contrairement aux reliquaires, bien des objets ne sont pas identifiables comme éléments du culte des saints. Certains s'offrent à des usages variés, et les brancards employés pour la procession des reliques serviront aussi lors de la Fête-Dieu. D'autres ne pourront être associés aux reliques qu'à l'issue d'un travail documentaire et rien ne différencie une statue en plâtre achetée pour une relique de sainte Philomène, de la simple statue de dévotion placée dans une église.

Le fonctionnement cultuel des reliques lors des deux derniers siècles apparait d'une complexité telle qu'il semble impossible de proposer une classification formelle ou fonctionnelle du mobilier. Plutôt que de chercher l'impossible définition du reliquaire, mieux vaut renverser la question et s'interroger sur la nature des éléments relatifs au culte des reliques.

\section{Comment inventorier le culte des reliques?}

Le responsable de l'inventaire ou du récolement d'une église s'interrogera sur ce qu'il convient de noter. On relève en général sur les reliquaires les éventuels marques ou poinçons, les principales représentations et le nom des saints, on y ajoute la désignation usuelle et la date estimée de fabrication ${ }^{15}$. Le grand nombre d'objets d'une incontestable variété formelle bien que souvent fabriqués en série, leur qualité esthétique souvent faible, expliquent les hésitations, les écarts sensibles dans les différentes notices, ainsi que la rareté des travaux de référence (fig. $\mathbf{n}^{\circ} 5$ ). 


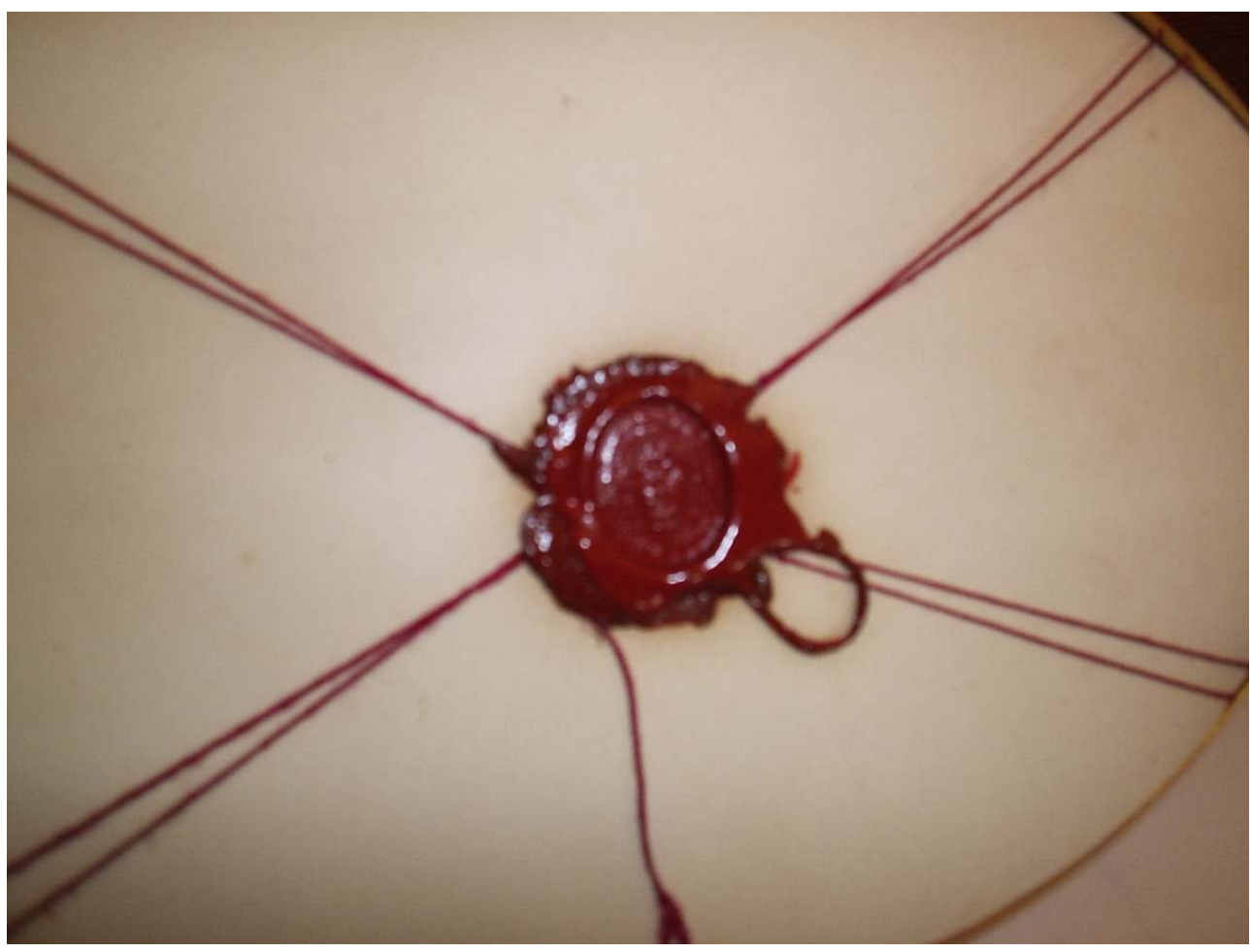

Sceau au dos d'un reliquaire. Sans sa présence, le culte des reliques est interdit. II faut en principe le rompre ou couper les fils pour accéder à la relique et cette intervention exige la présence de l'ordinaire du lieu, seul habilité à remettre un sceau.

Phot. Y. Gagneux. (c) Y. Gagneux.

Peintures, sculptures ou vitraux sont également inventoriés, mais leur lien éventuel avec les reliques n'apparaît pas toujours dans la structuration des données. De même, la recherche des états successifs est presque toujours négligée parce qu'elle suppose un travail rarement compatible avec les exigences pratiques d'un inventaire, et pour un résultat aléatoire. Pourtant, une démarche scientifique suppose la connaissance de l'histoire des œuvres.

Dès lors qu'il ne s'agit plus de traiter objet après objet mais de s'intéresser aux manifestations cultuelles suscitées par les reliques, il est inévitable que d'autres besoins apparaissent.

\section{Relevé des représentations}

Considérons dans un premier temps les représentations. Il est d'usage de noter les inscriptions, les représentations figuratives, les sceaux, les blasons, on peut même indiquer les couleurs lorsqu'un symbolisme est susceptible d'y être attaché, notamment la couleur du tissu sur lequel sont posées les reliques ${ }^{16}$.

Les sceaux doivent, selon les prescriptions de l'Église, interdire l'accès direct aux reliques et il ne saurait être question de les rompre, sans quoi la validité même de la relique s'en trouverait atteinte. Généralement en cire rouge, ils se trouvent au dos des petits reliquaires et, pour les reliquaires en bronze ou laiton doré, sous le couvercle ou dans la base $^{17}$. Lorsque de petits reliquaires sont placés dans un autre lui-même scellé, ils 
deviennent inaccessibles et il faut se résoudre à ne pas voir leurs sceaux (fig. $\left.\mathbf{n}^{\circ} \mathbf{6}\right)$. Tous ces cachets ne sont pas lisibles, soit qu'ils aient été mal imprimés, soit que le couvercle placé sur la cire encore molle en ait écrasé le motif. Chaque évêque et archevêque dispose de ses propres armoiries que des manuels d'héraldique aident à identifier ${ }^{18}$, on trouve aussi les sceaux de prélats étrangers ou de dignitaires d'ordres religieux.

Figure 6

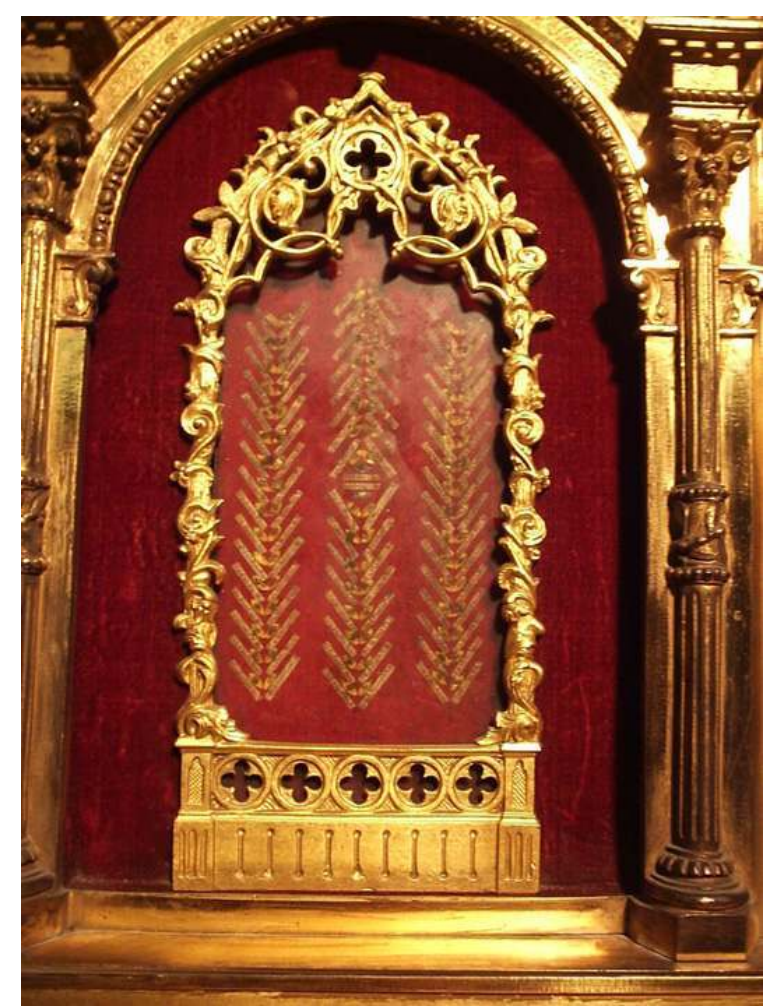

Détail d'un calendrier reliquaire. Paris, Notre-Dame-des-Victoires. De nombreuses reliques proviennent de martyrs particulièrement obscurs mentionnés seulement dans des hagiographies très spécialisées. Les indications des noms n'ont pas ici pour but d'individualiser les saints et l'on peut s'interroger sur l'utilité de relever ces indications.

Phot. Y. Gagneux. (c) Y. Gagneux.

Les sceaux suppléent souvent à la disparition des authentiques ${ }^{19}$ car ils subsistent sur presque tous les reliquaires. Les boîtes les plus petites servent au transport des reliques plus qu'à leur vénération et font rarement l'objet d'une visite ultérieure, aussi le sceau donne-t-il la provenance géographique ainsi que des indications chronologiques sur la diffusion de la relique. Les reliquaires plus importants sont fréquemment acquis sur le lieu même de vénération et l'on peut donc apprendre sous quel prélat les reliques ont été disposées ou vérifiées pour la dernière fois, à quelles dates les reliques des saints coutumiers ont été de nouveau présentées aux fidèles après la Révolution. À grande échelle, le relevé des sceaux souligne le rôle des différents diocèses dans la reconstitution des trésors de reliques après la Révolution, renseigne sur la diffusion des reliques, leur étendue, l'importance de certains ordres dans l'expansion des reliques d'un saint. Les sceaux sur les reliques de saints proclamés au $\mathrm{XIX}^{\mathrm{e}}$ ou au $\mathrm{XX}^{\mathrm{e}}$ siècle révèleront par exemple l'activité d'un couvent dont un membre aura été élu, et montreront si la diffusion coïncide avec la béatification, la canonisation, ou si elle s'inscrit dans la durée. 
Les travaux de Arnold Van Gennep ont démontré l'intérêt de relever le nom du saint accompagnant presque toujours la relique ${ }^{20}$ et il est pertinent, sur une zone géographique importante, de chercher les vestiges de saints ayant eu un rayonnement local ou régional - travail qui suppose une certaine connaissance historique préalable.

Plus les reliques sont regroupées, moins les transcriptions sont systématiques : on est d'ailleurs fondé à s'interroger sur l'utilité de cet exercice lorsqu'une centaine de fragments voisinent, quand le clergé rassemble par commodité toutes les reliques dans un seul reliquaire, ou lorsqu'il constitue des calendriers avec une parcelle de saint pour chaque jour de l'année (fig. $\mathbf{n}^{\circ} \mathbf{7}$ ). Certes le rapprochement fait parfois sens : saints issus de familles nobles, dont l'action offre des similitudes, appartenant à un même ordre. Faut-il pour autant noter "reliquaire de Franciscains ", " de Pères de l'Église », sans donner le détail ? Doit-on signaler les reliques qu'une place centrale, des rapprochements d'ordre visuel (symétrie; couleur des fonds...) dégagent d'un vaste regroupement ? La présentation ne joue-t-elle pas un rôle déterminant?

Figure 7

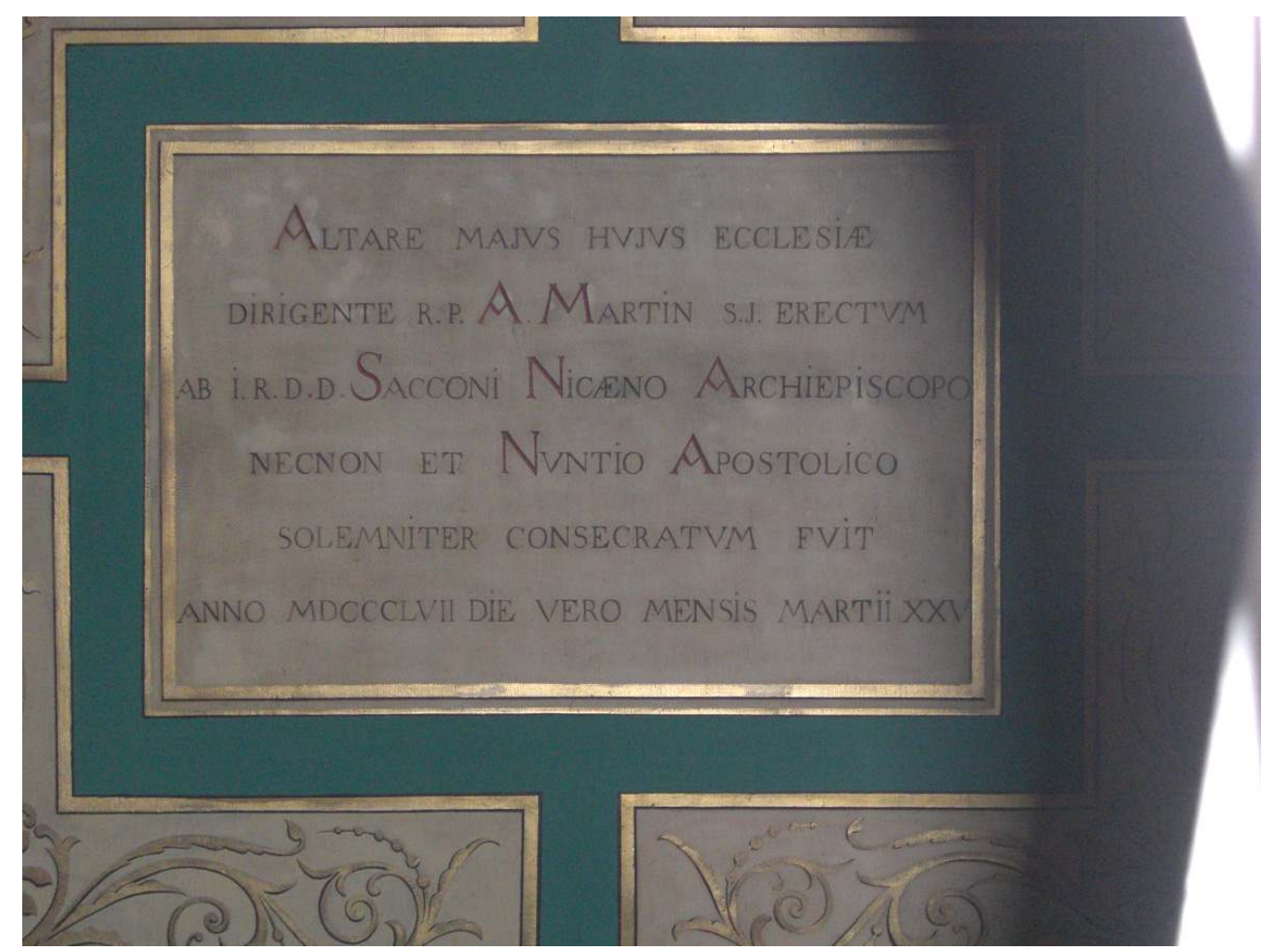

Plaque indiquant le nom de l'architecte du monument supportant la châsse de saint Vincent de Paul. La présence d'une telle indication est exceptionnelle, l'anonymat étant la règle. Paris, chapelle des Lazaristes.

Phot. Y. Gagneux. (c) Y. Gagneux.

Il convient de porter intérêt à la forme même de l'écriture. Celle-ci comporte parfois des recherches décoratives qui ne sont pas toujours seulement esthétiques -l'écriture gothique renverra au Moyen Âge, ce qui n'est pas neutre au XIX ${ }^{\mathrm{e}}$ siècle - ; le choix de la langue n'est pas sans incidence puisque le latin "cléricalise » la relique; l'inscription renseigne sur l'univers mental de celui qui la rédige (mettre « sainte Thérèse » sans autre précision ne permet qu'aux initiés de savoir quelle sainte est honorée, la française ou 
l'espagnole); les indications hagiographiques existent, même sommaires (B. pour bienheureux, S. pour saint ${ }^{21}$, V. pour vierge ; M. pour martyr...).

Quand bien même le relevé des inscriptions pourrait être plus poussé, il reste pratique courante. En revanche, les authentiques, ces documents étroitement liés au culte puisque la légitimité de la relique exige à la fois leur présence et celle du sceau, sont presque toujours oubliés comme si la séparation matérielle entraînait une dissociation cultuelle. L'authentique se présente en général comme une grande feuille partiellement imprimée, aux armoiries de celui qui inspecte la relique (souvent l'Ordinaire du lieu), que l'on range souvent à part, mais qu'on trouve aussi parfois dans le reliquaire, par exemple sous le toit ou dans la base (fig. $\left.\mathbf{n}^{\circ} \mathbf{8}\right)$. Signée par un dignitaire ecclésiastique, elle est complétée par des indications sur la nature, la provenance des vestiges, et la date de leur reconnaissance. Le lien n'est pas toujours simple à établir avec la relique : celle-ci peut avoir perdu son authentique ou, au contraire, l'authentique aura survécu à la disparition des reliques. Plusieurs actes concernent parfois une seule relique, puisqu'il faut renouveler l'attestation à chaque rupture des sceaux, et que les pièces antérieures sont rarement détruites.

Figure 8

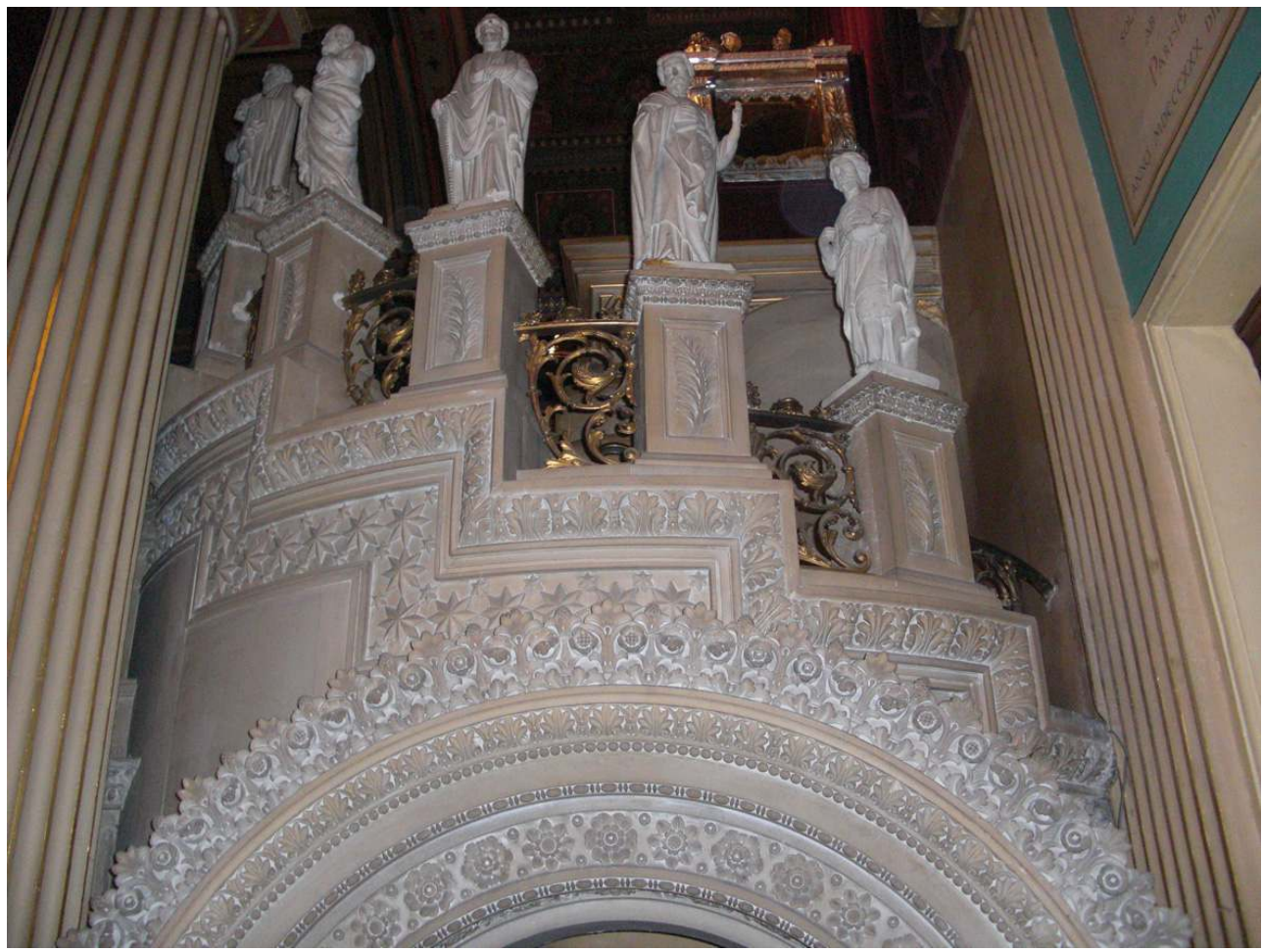

Statuettes d'apôtres dominant l'escalier conduisant à la châsse de saint Vincent de Paul. Paris, chapelle des Lazaristes. On distingue la châsse en haut à droite. De telles statuettes seront inventoriées mais pas toujours mises en relation avec les reliques du saint, alors que leur juxtaposition avec le corps de saint Vincent de Paul obéit à une conception iconographique complexe et raisonnée.

Phot. Y. Gagneux. (c) Y. Gagneux.

Intégrer de tels documents dans l'inventaire implique une modification des bases de données, qui ne seraient plus seulement composées d'une série d'objets, mais proposeraient des liens vers des références éventuellement conservés hors de l'édifice ${ }^{22}$. 
À Paris, l'ampleur de la représentation est rare. Exceptionnelles sont les chapelles où la présence de reliques détermine le décor monumental, comme celle dédiée à sainte Philomène à Saint-Merry, qu'ornent des peintures d'Amaury-Duval (fig. $\left.\mathbf{n}^{\circ} \mathbf{9}\right)$. Le plus souvent, l'image occupe la face d'une petite boîte en bronze et relève du stéréotype (Sacré-Cœur, Vierge de douleur, portrait de Vincent de Paul...) sans rapport avec le contenu. L'opportunité de relever des motifs généraux comme la croix, des ornements végétaux ou architecturaux, n'apparaît pas toujours. Mais si le Sacré-Cœur associé à sainte Geneviève n'aura aucun sens, il en prendra un avec une relique de MargueriteMarie Alacoque, puisque cette sainte a largement contribué à en établir la dévotion ${ }^{23}$ (fig. $\left.n^{\circ} 10\right)$.

Figure 9

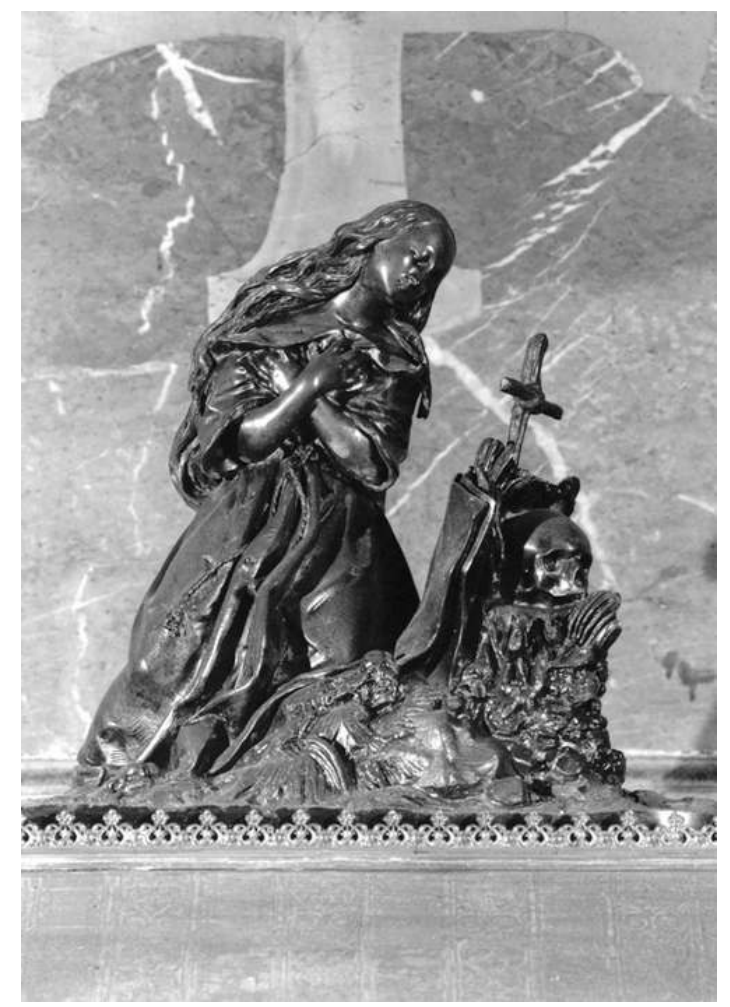

Auguste Préault et François-Désiré Froment-Meurice, effigie de sainte Madeleine repentante pour le reliquaire de sainte Madeleine, bronze argenté. Paris, église Sainte-Marie-Madeleine. Un exemple rare d'une réalisation véritablement artistique associée à des reliques. Cette œuvre est d'une qualité exceptionnelle mais la plupart des reliquaires parisiens proviennent d'orfèvres industriels vendant sur catalogue des productions en bronze doré ou en laiton. Les reliques peuvent aussi être placées dans de simples médaillons voire collées sur de petites cartes en papier. Les églises conventuelles conservent parfois des quantités considérables de tels objets. Ce nombre et cette diversité ne prêchent pas toujours en faveur d'un inventaire systématique.

Phot. Jean-Marc Moser (c) COARC/Parisienne de photographie. 
Figure 10

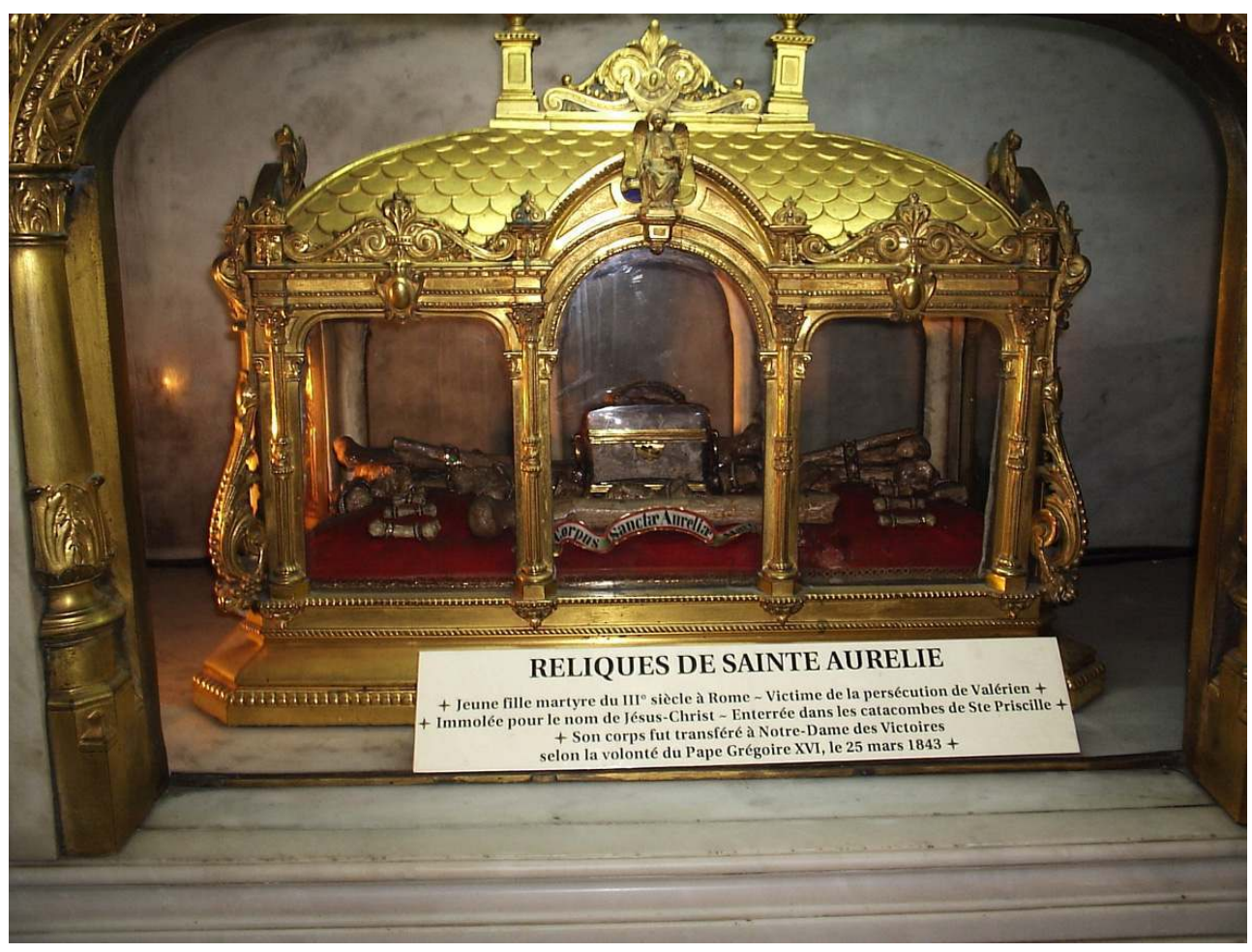

Reliquaire de sainte Aurélie. Paris, Notre-Dame-des-Victoires. Un exemple d'évolution de la présentation. Le premier reliquaire présentait sous l'autel une effigie en cire, détruite lors de la Commune, qu'a remplacé ce nouveau reliquaire en métal. Plus récemment, la paroisse a jugé utile de poser un panneau donnant quelques indications sur la biographie de sainte Aurélie.

Phot. Y. Gagneux. (C) Y. Gagneux.

De manière générale, l'iconographie liée aux reliques reste pauvre. Sans rendre compte de la complexité des installations, les représentations sont pourtant presque toujours notées lors d'un inventaire.

\section{Relevé de l'identité sociale}

Il en va tout autrement pour ce qui définit socialement le saint et que l'on néglige toujours. Omission paradoxale car la relique se caractérise par sa capacité à établir une relation entre le fidèle et le saint. Elle est en effet non seulement présence réelle puisque vestige physique (reliques corporelles) mais aussi présence sociale, et elle participe à ce titre au jeu des distinctions que l'homme établit sans cesse avec ses congénères, opposant saint et non saint, jeune ou vieux, homme ou femme, local ou étranger, etc. Ces dissociations peuvent être dites, représentées, mais aussi matérialisées par l'ensemble des traitements qui témoignent de la considération. Elles ne font pas nécessairement ressortir un individu et dégagent parfois les caractéristiques d'un groupe, voire tendent au concept. Enfin, la plupart de ces aménagements ont une efficacité relative : une châsse secondaire dans une grande cathédrale prendra des dimensions colossales dans une petite chapelle ; la notion de luxe varie selon la richesse de la communauté.

Les marques de respect ne sont pas nécessairement visibles, ainsi la mise en ordre des ossements, l'utilisation de fil doré pour fixer les reliques, ou d'une soie particulièrement riche pour les couvrir (la soie est exigée par la liturgie pour envelopper les corps des 
serviteurs de Dieu). L'usage de vêtir les reliques trouve en France au XIX ${ }^{\mathrm{e}}$ siècle un prolongement très particulier avec l'expansion des effigies en cire associées dans un premier temps aux corps saints issus des catacombes, avant d'être étendues aux saints récemment canonisés (fig. $\mathbf{n}^{\circ} \mathbf{1 1}$ ). Ces figures sont en effet pourvues d'habits similaires à ceux des vivants, sans pour autant reconstituer l'habit porté par le saint avant sa mort. Celui qui aura fait vœu de pauvreté sera couvert de soie et d'or, conformément à sa position dans l'Église.

\section{Figure 11}

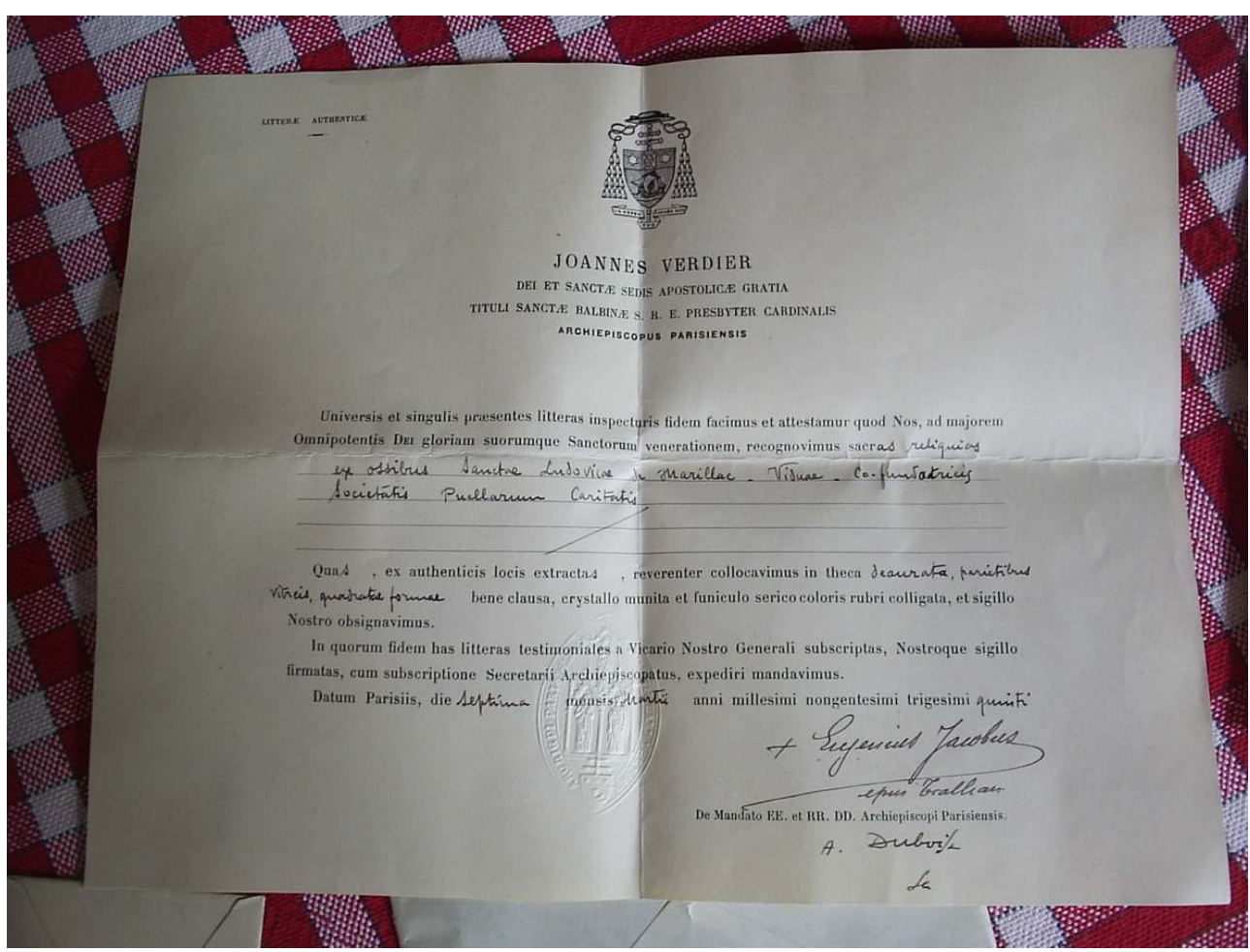

Authentique de reliques de sainte Louise de Marillac. Paris, église Saint-Laurent. Ces documents devraient être systématiquement associés aux reliques. Mais on trouve souvent des reliquaires sans authentiques ou des authentiques sans reliquaire. Chaque fois que le reliquaire est ouvert, un nouvel acte doit être établi. Plusieurs authentiques séparés par le temps peuvent ainsi concerner la même relique.

Phot. Y. Gagneux. (C) Y. Gagneux.

Le traitement des reliques ne reproduit en effet pas la vie des saints. La modestie du curé d'Ars aurait-elle souffert l'exposition permanente au milieu du chœur, dans une châsse richement ornée ${ }^{24}$ ? Et que dire de l'importance accordée dans la chapelle de la Médaille miraculeuse à Catherine Labouré, la "sœur du poulailler », ainsi nommée en raison des seules tâches humbles qu'elle parvenait à exécuter ? Il ne s'agit pas de restituer au saint le cadre de son existence terrestre mais de montrer sa nouvelle place au sein de l'Église (fig. $\mathbf{n}^{\circ}$ 12). 
Figure 12

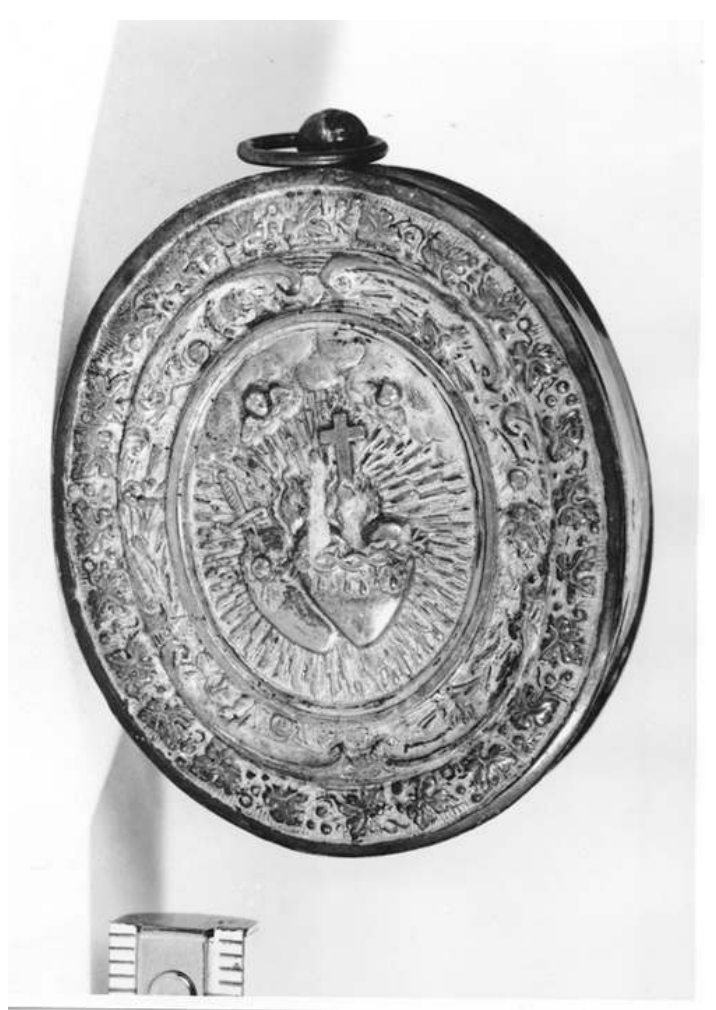

Dos d'un reliquaire. Un exemple de ces représentations très banales puisque appliquées de manière industrielle. Le Sacré Cœur, la Vierge de l'Immaculée Conception, la Croix... forment des motifs assez répandus et généralement sans lien direct avec les saints dont le médaillon renferme les reliques.

Phot. Y. Gagneux. (c) Y. Gagneux. la fois présence réelle du saint et propriété de l'église. Les traitements de conservation, la prévention du vol, l'implantation des sceaux doivent concilier déférence et impératifs sécuritaires. De gros barreaux en fer opposeraient une excellente défense contre le vol mais sembleraient emprisonner le saint. De même, le bocal d'alcool ou de formol, associé aux échantillons d'histoire naturelle, ne témoigne pas d'un respect suffisant et ne s'emploie jamais pour les reliques, mêmes carnées. Le traitement très poussé dont a bénéficié le corps de sainte Roseline de Villeneuve, traité avec beaucoup de soin dès 1894, et soumis en 1995 à un traitement de conservation préventive exceptionnel ${ }^{25}$, n'a aucun équivalent à Paris. malgré la fréquence des processions au XIX ${ }^{\mathrm{e}}$ siècle. Les reliques sont alors mises à l'honneur avec le matériel (des brancards) utilisé pour la Fête-Dieu ou la procession de statues.

revanche apparait essentiel ce qui définit socialement la relique dans le «logement », par exemple la richesse ou la qualité du travail de décoration, la place plus ou moins importante accordée au saint, la proximité ou non avec d'autres saints, Dieu ou les fidèles. On compte à Paris peu d'installations monumentales comme celles bâties à Reims, Tours ou Ars-sur-Formans ${ }^{26}$. La chapelle des Lazaristes qui conserve le corps de saint Vincent de Paul ou la chapelle Sainte-Geneviève à Saint-Étienne-du-Mont occupent un espace 
exceptionnellement vaste. Quelques églises vouent une chapelle au saint dont elles possèdent les reliques ; ailleurs, l'espace dédié est souvent limité au seul reliquaire.

Le choix du lieu est marque d'égard pour un saint quand on place ses reliques dans un endroit familier, ainsi Catherine Labouré présentée dans l'autel où lui apparut la Vierge, ou la crypte qui abrita longtemps le corps de saint Merry et où l'on voulut, pour cette raison, remettre les reliques. Les emplacements ne se valent pas tous et comme l'église accueille de nombreux occupants (présence divine mais aussi clergé, fidèles, personnages figurés par des statues ou des vitraux, morts inhumés dans l'édifice) les priorités trahissent l'établissement implicite d'une hiérarchie, variable selon les lieux et les temps.

L'habitat fonctionne comme celui des vivants. En particulier, le regroupement implique des relations de voisinage, et intègre des critères variés qu'il est intéressant de dégager, l'assemblage des reliques étant significatif de l'état d'esprit de l'ordonnateur - plus que de celui des fidèles auxquels s'offre rarement la possibilité d'examiner le détail d'objets souvent présentés de loin et soumis à un éclairage inégal.

À Paris, les principaux critères de rapprochement sont géographiques, chronologiques, familiaux ou professionnels. Dans les regroupements importants, les individualités s'estompent au profit de caractères généraux. On trouvera le cortège des apôtres (selon des configurations variables tant la liste des apôtres prête à confusion), la sainte Famille, des confesseurs, des Dominicains... (fig. $\mathbf{n}^{\circ} \mathbf{1 3}$ ) Ce sont aussi des vertus que le regroupement de vierges ou de martyrs propose en exemple. La disposition ne tient pas compte de la réalité historique et des saints ne s'étant jamais croisés de leur vivant voisinent selon une logique propre aux $\mathrm{XIX}^{\mathrm{e}}$ ou $\mathrm{XX}^{\mathrm{e}}$ siècles. Ainsi saint Pierre et sainte Geneviève dont la réunion, dans le principal reliquaire de l'église catholique gallicane Sainte-Rita, constitue un véritable manifeste ${ }^{27}$. 


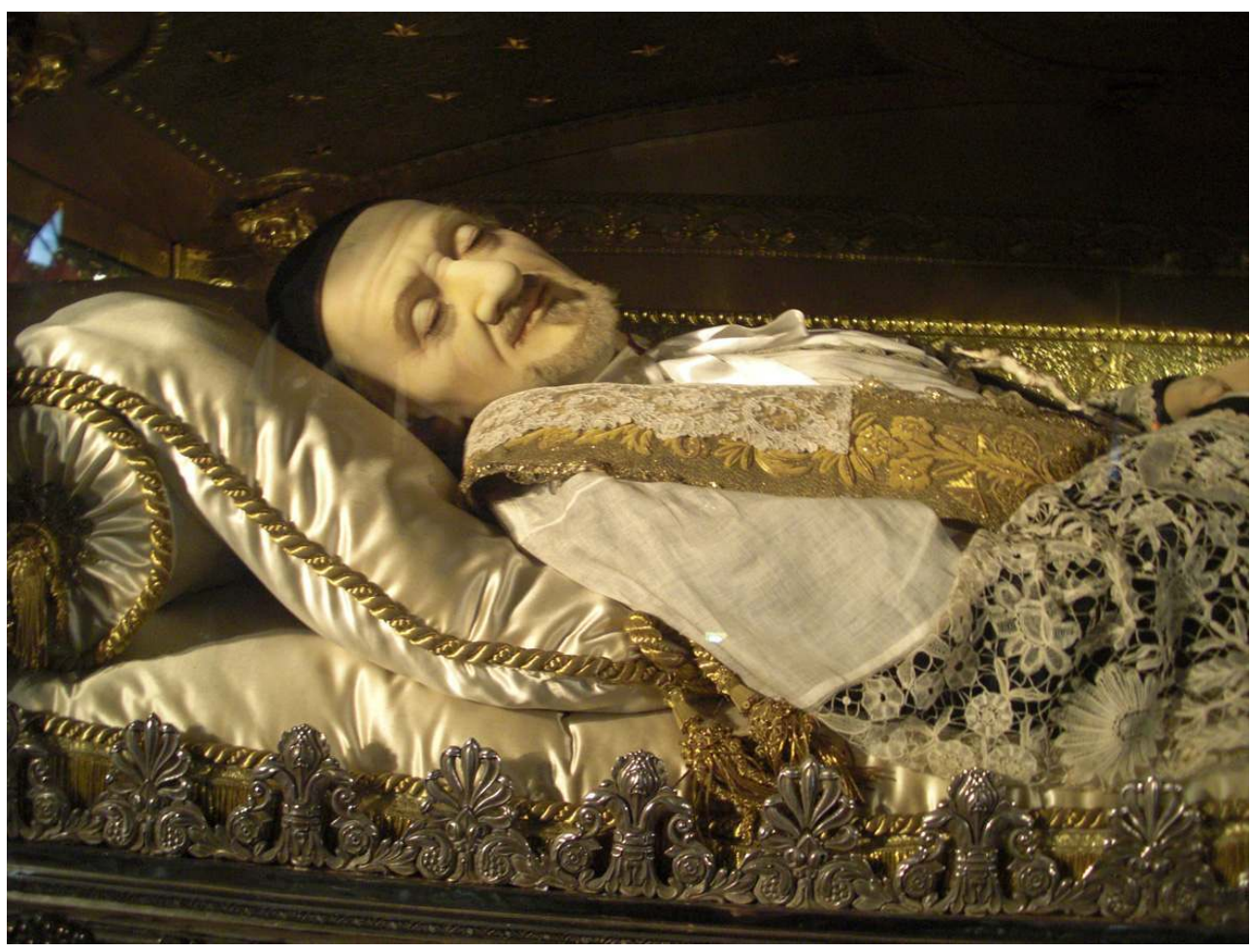

Corps de saint Vincent de Paul. Paris, chapelle des Lazaristes. Exemple particulièrement spectaculaire d'habillage des reliques, avec un masque et des mains de cire ainsi que des habits sacerdotaux. La richesse de ce vêtement, comme celle de l'oreiller et des coussins en soie brodée d'or, n'est pas une tentative pour restituer le costume que portait Vincent de Paul de son vivant : elle indique son importance dans l'Église catholique. On sait que saint Vincent de Paul est non seulement le fondateur de la congrégation de la Mission mais aussi un des modèles de prêtres pour l'Église catholique.

Phot. Y. Gagneux. (c) Y. Gagneux.

Un saint dont les reliques, même rares, sont souvent seules ou occupent une position privilégiée dans un regroupement, sera plus honoré qu'un autre dont les reliques seraient plus abondantes mais réparties au milieu d'autres vestiges. Ainsi, le nombre de reliques atteste l'intérêt porté à la diffusion des restes d'un saint mais n'indique pas à lui seul le degré de vitalité du culte. À Paris, vingt églises possèdent trente-six reliques de sainte Anne, mais toujours placées avec au moins cinq autres reliques, ou dans des boîtes si petites qu'elles ne sont pas destinées au culte. Inversement, les vingt-huit reliques de Jean-Marie Vianney sont neuf fois mises à l'honneur dans des reliquaires de grande dimension, où elles se trouvent seules ou avec un ou deux saints au plus.

43 L'inégalité des traitements, indépendamment des contraintes matérielles (l'espace ou l'argent disponible par exemple) met en évidence une hiérarchie évoluant selon les lieux ; on tiendra en général saint Augustin en plus grande estime qu'un saint dont la renommée ne dépasse pas les limites d'un diocèse, sauf dans ledit diocèse ou la hiérarchie sera inversée.

44 C'est donc la conception ecclésiastique de l'histoire de l'Église qui apparaît dans le choix et la disposition des reliques, une histoire qui ne coïncide pas nécessairement avec celle que présentent les écrits contemporains des reliquaires: il existe parfois une dualité entre ce qui se dit et ce qui se fait, et bien des choses qui transparaissent dans l'agencement des reliques - la hiérarchie parmi les saints, la définition du collège des 
apôtres, l'éloge de la virginité féminine - ne trouvent guère d'équivalent direct dans les écrits contemporains, qu'il s'agisse des semaines religieuses ou des bulletins paroissiaux.

\section{Le culte}

Outre la définition du saint, les «installations » organisent le culte, soit qu'elles y incitent, soit qu'elles le prolongent, avec les prières imprimées à l'attention des fidèles, les porte cierges, les sièges ou prie-dieu placés devant la relique, l'organisation d'un parcours, les ex-voto, les cierges... Hormis les plaques en marbres placées sur les murs, la plupart de ces éléments sont mobiles ou présentent un caractère éphémère, et il n'en reste pas toujours de trace matérielle (fig. $\left.\mathbf{n}^{\circ} \mathbf{1 4}\right)$.

\section{Figure 14}

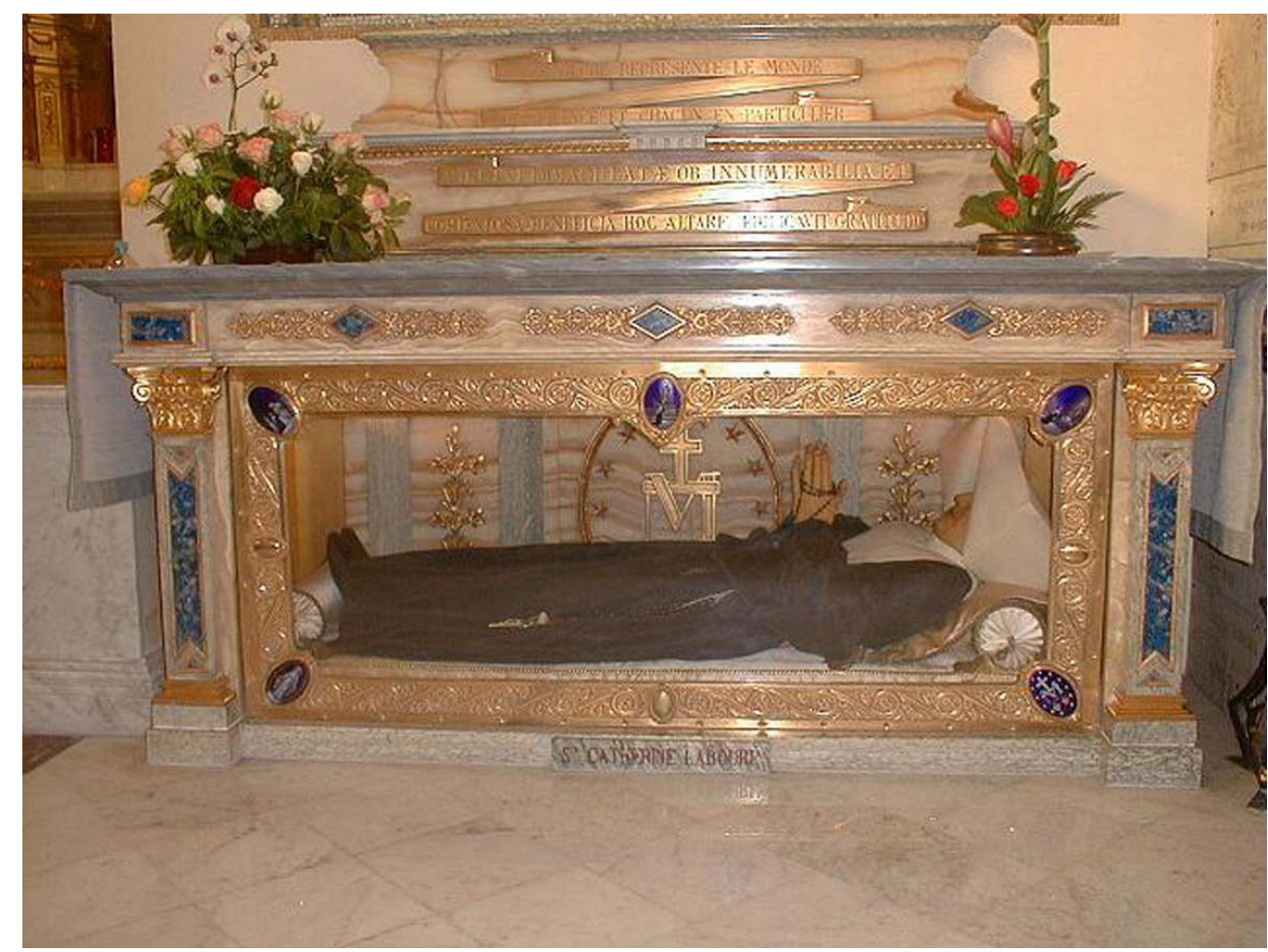

Corps de sainte Catherine Labouré. Paris, chapelle de la Médaille miraculeuse. L'emplacement de la relique (à la place même de l'autel où lui apparut la Vierge Marie) contribue non seulement à qualifier sainte Catherine Labouré mais aussi à donner à son effigie une importance considérable. Il faut également souligner la proximité du corps de sainte Louise de Marillac première supérieure des Filles de la Charité et du cœur de saint Vincent de Paul, fondateur de cet ordre.

Phot. Y. Gagneux. (C) Y. Gagneux.

Les installations favorisant le contact se montrent davantage pérennes. Cette pratique est admise par l'Église mais souvent présentée au XIX siècle à Paris comme un usage ancien, même si l'observation des objets atteste sa permanence. Ce sera la fenêtre aménagée sur la châsse de sainte Geneviève (fig. $\mathbf{n}^{\circ} \mathbf{1 5}$ ) pour permettre le contact direct avec la pierre du tombeau, ou les baiser de paix renfermant des reliques. Un tel culte exige un compromis entre le quant-à-soi du saint et la ferveur des fidèles. 
Figure 15

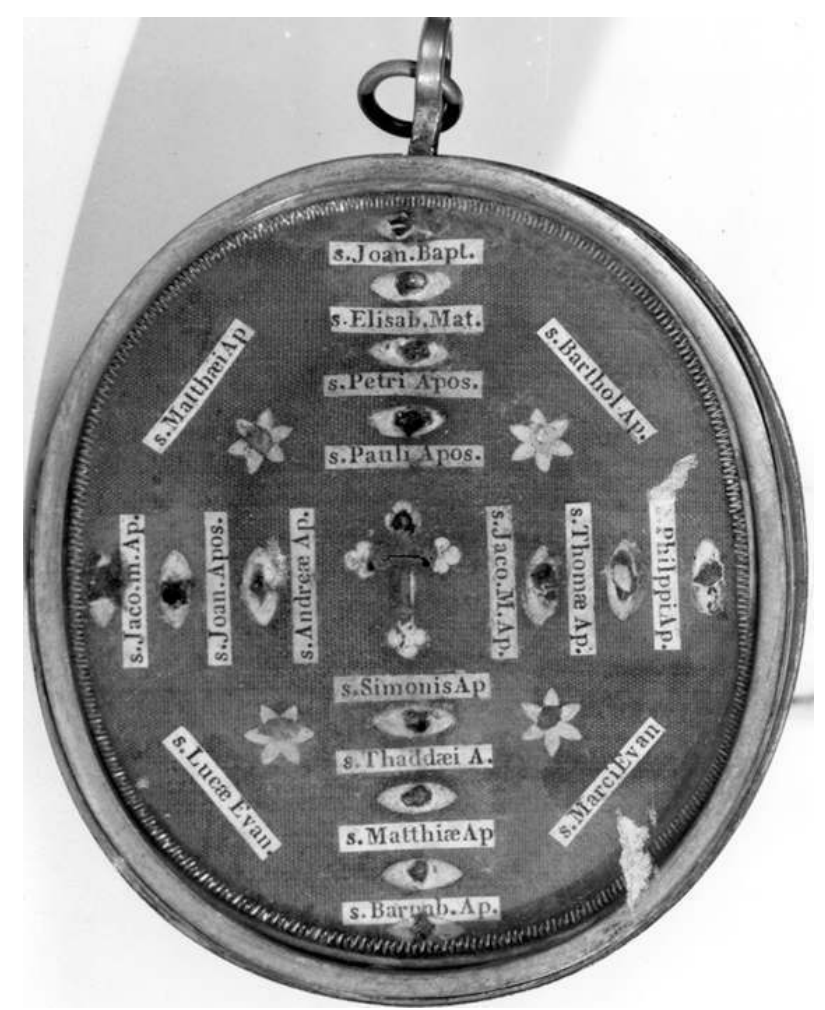

Reliquaire de saints apôtres. Paris, église Saint-Laurent. Un exemple de ces regroupements qui dégagent une caractéristique collective. Ce reliquaire présente l'apostolicité. On notera que les apôtres sont ici quatorze et que quatre saints contemporains du Christ les ont rejoints.

Phot. Emmanuel Michot. (C) COARC/Parisienne de photographie.

Il en va de même avec la relation visuelle, essentielle à Paris lors des deux derniers siècles puisque sur plus de 800 reliquaires, seulement trois dissimulent entièrement les reliques.

Le culte s'exprime enfin de manière détournée, lorsque des usages supposés antiques sont adaptés, tel le passage sous la châsse que l'on autorise dans la chapelle des Lazaristes, non plus au prix d'une éprouvante reptation mais en aménageant un escalier qui passe sous la châsse (fig. n¹6). 
Figure 16

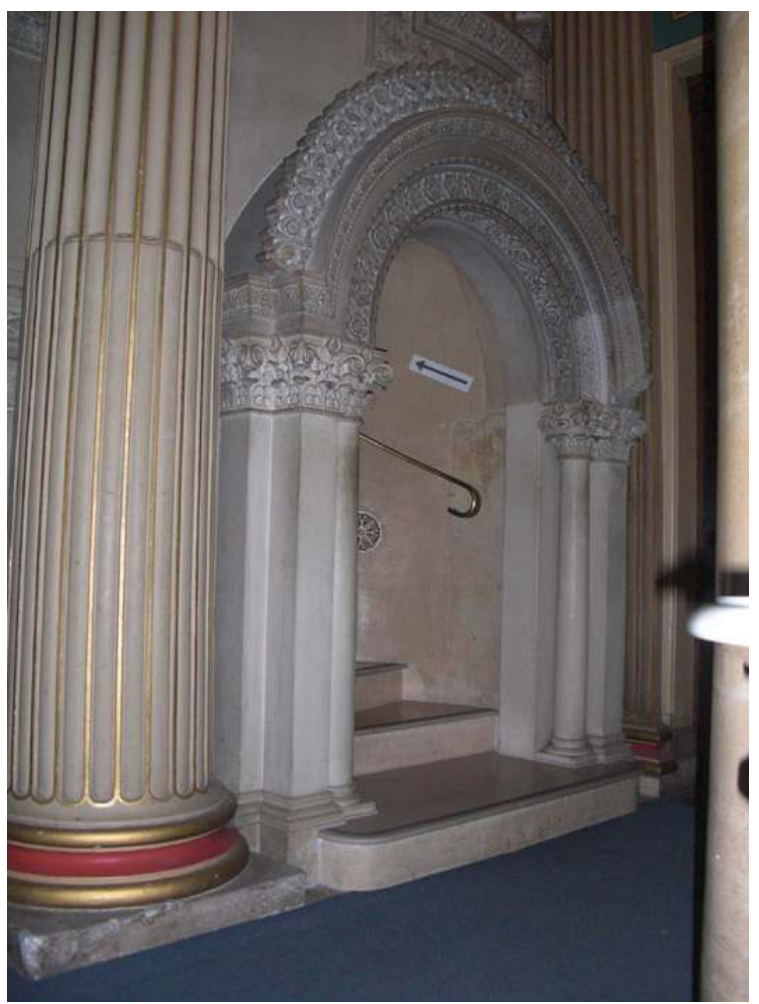

Escalier conduisant à la châsse de saint Vincent de Paul. Paris, chapelle des Lazaristes. Un exemple de circuit conditionnant le culte. II faut rentrer chez le saint, se donner le mal de monter plusieurs marches pour voir la châsse. Le contraste entre l'obscurité de l'escalier et l'espace largement éclairé où se trouve le corps de Vincent de Paul contribue à souligner l'importance du saint.

Phot. Y. Gagneux. (c) Y. Gagneux.

\section{Données documentaires}

La connaissance des décors éphémères et de l'ordonnance des cérémonies complète l'étude des installations permanentes, notamment pour les reliques les plus honorées, d'autant plus que l'apparat déployé lors des grandes cérémonies du XIX ${ }^{e}$ siècle était de nature à marquer durablement les esprits. La connaissance de l'expression du culte exige non seulement de relever les objets et leur rapport à l'édifice, mais aussi d'identifier les auteurs, de retrouver les dates et les lieux d'exécution (fig. $\mathbf{n}^{\circ} \mathbf{1 7}$ ). 


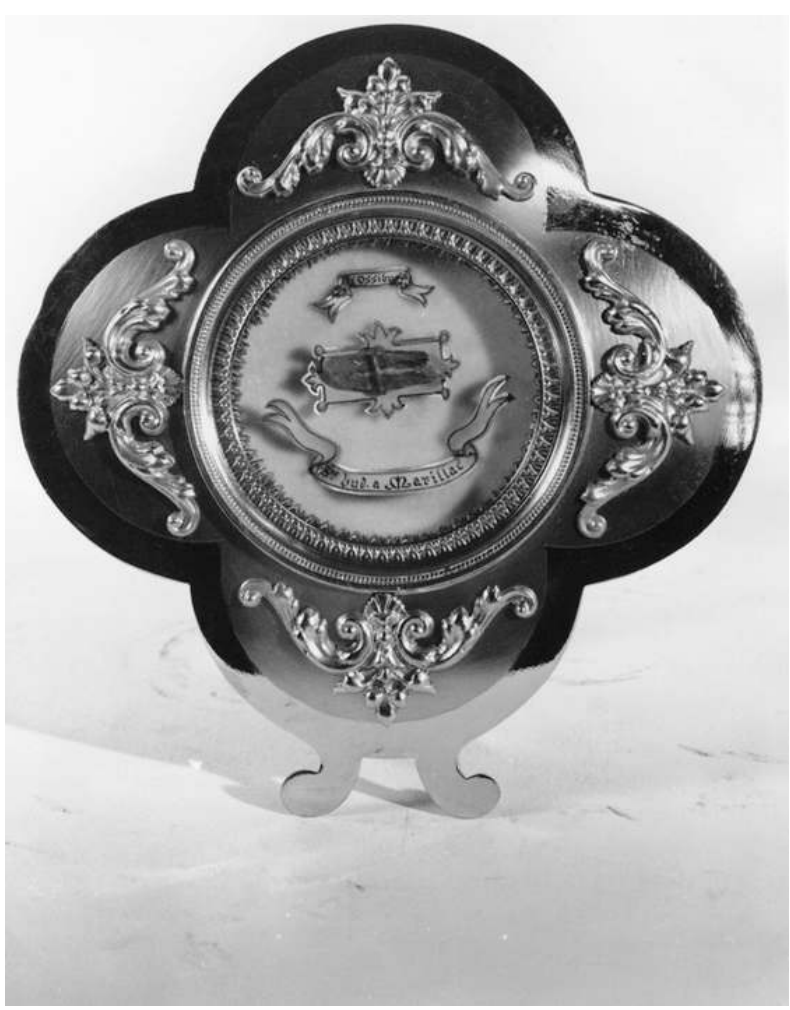

Baiser de paix reliquaire. Paris, église Saint-Laurent. Un exemple de culte par contact direct. Une poignée permet de présenter cet objet aux fidèles qui embrassent la vitre derrière laquelle se trouve la relique.

Phot. Emmanuel Michot. @ COARC/Parisienne de photographie.

Les reliquaires font intervenir plusieurs auteurs ; celui qui conçoit la pièce, l'orfèvre qui la réalise, le prêtre qui en aménage l'intérieur, fixe les reliques, rédige les inscriptions, les services diocésains qui remplissent les authentiques. Il faut ajouter les intervenants sur les autres éléments liés au culte comme les peintures murales, les statues, le mobilier, etc. En ce qui concerne les boîtes en métal, le nom du fabricant ne se trouve qu'exceptionnellement sur des objets d'une qualité souvent secondaire. Peu de reliquaires sont en métal précieux et donc sans poinçon; on possède en revanche des catalogues de fabricants très utiles pour l'identification de l'orfèvre ${ }^{28}$, même si le commanditaire demande parfois qu'on apporte des modifications aux modèles, afin de disposer d'un objet a priori unique - de telles pratiques sont mises en évidence à la fin du XIX ${ }^{\mathrm{e}}$ et au début du XXe siècle par les archives de Sainte-Clotilde de Reims (fig. $\left.\mathbf{n}^{\circ} \mathbf{1 8}\right)$. 
Figure 18

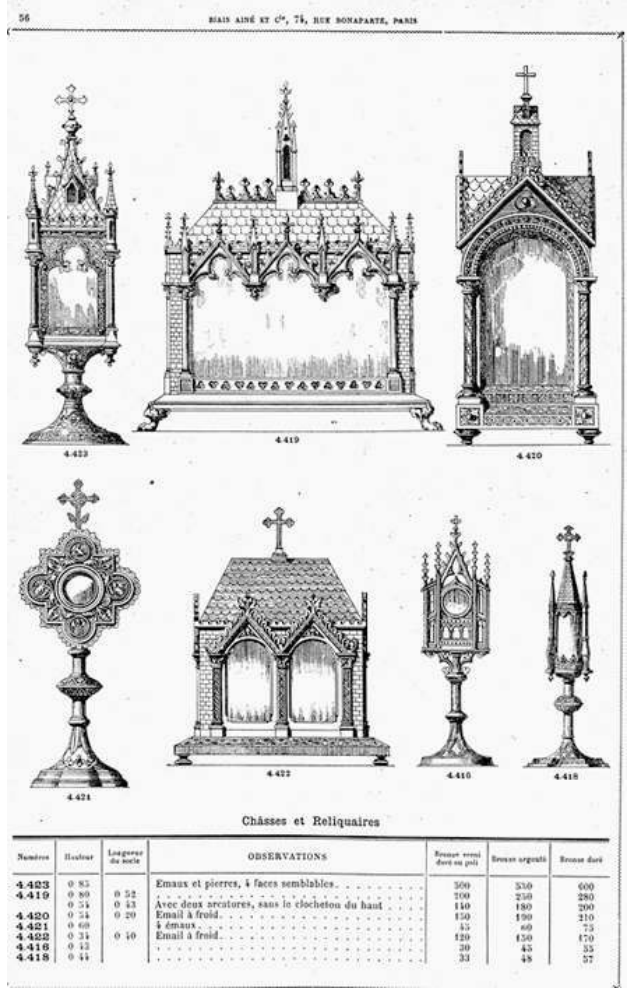

Catalogue de l'orfèvre Biais, p. 56. Un exemple de ces nombreux catalogues proposant un choix étendu de productions semi-industrielles à l'usage des églises.

Phot. Y. Gagneux. (c) Y. Gagneux.

Est-il pour autant opportun d'identifier l'intégralité des intervenants? Si l'article sur l'orfèvrerie publié dans un précédent numéro d'In situ montre combien le relevé des orfèvres devient fécond, dès lors qu'on s'intéresse à la diffusion des pièces, donc à l'économie des objets liturgiques ${ }^{29}$, qui étudiera la création des pique-cierges ? (fig. $\mathbf{n}^{\circ}{ }^{19}$ ) 


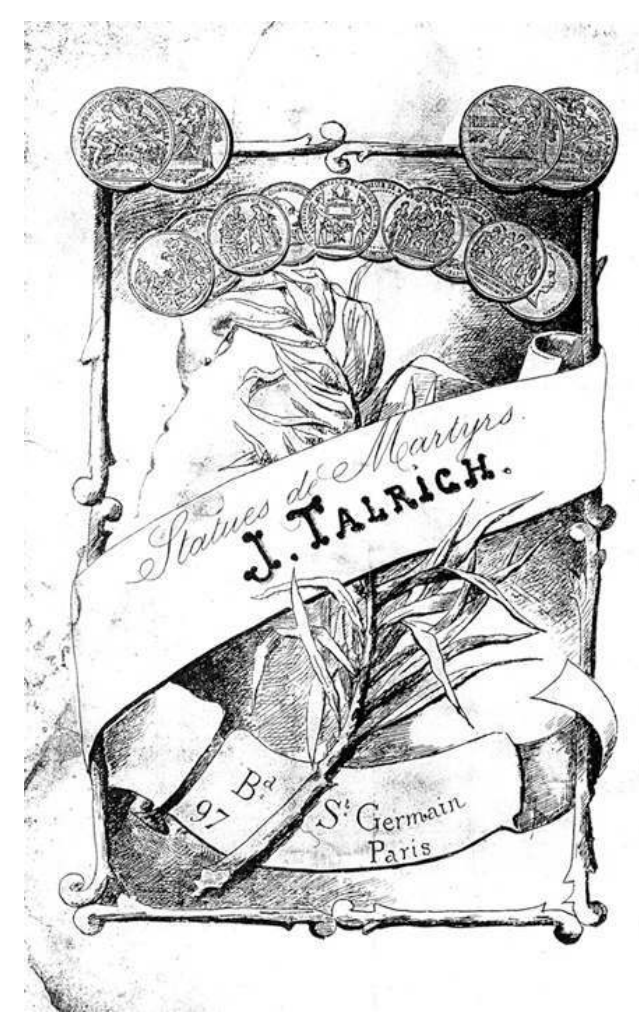

Page de couverture d'un catalogue de Jules Talrich. Archives de l'église Sainte-Clotilde, Reims. Un catalogue beaucoup plus rare, ne proposant que des figurines en cire. Jules Talrich, modeleur de la Faculté de médecine, devint dans la seconde moitié du XIXe siècle le spécialiste de ces représentations alors très à la mode.

Phot. Y. Gagneux. (c) Y. Gagneux.

Le même type de recherche s'applique aux lieux de fabrication, avec des réserves similaires sur l'intérêt de ces questions. Est-ce le rôle d'un service d'inventaire d'y répondre?

La manière de faire enfin, qu'il s'agisse de la nature de l'agencement ou de la façon dont on le réalise, relève de l'analyse sociale. Le style marque historiquement les œuvres et donne de ce fait des indications sur le contexte de leur fabrication. L'utilisation de références médiévales pour les grands reliquaires en fait ainsi des armes dans la lutte opposant au milieu du XIXe siècle les tenants de l'obéissance au pape et ceux favorables à une indépendance de l'Église de France ${ }^{30}$. Mais parfois des objets néo-gothiques ont été utilisés sans connotation politique, et c'est l'étude seule qui détermine alors ce qui fait sens.

\section{Conclusion}

Le choix des éléments à relever lors d'un inventaire reflète l'idée qu'on se fait de l'objet, et les questions que l'on se pose: dans les faits, la grille d'analyse est presque toujours empirique. Pour qu'il en soit autrement, il faudrait faire appel à une théorie de l'objet, susceptible d'élargir considérablement le champ des questions et de développer les possibilités d'analyse. Pour autant, une théorie ne détermine que très lentement la recherche et ne dit pas, dans l'immédiat, quelles questions il convient de poser lors d'un 
inventaire. Son principal apport consiste à fédérer des modalités de recherches qui ne s'articulent pas toujours clairement les unes avec les autres (analyse stylistique, rôle économique, fonctions cultuelles, histoire du goût...). Au demeurant, depuis les tentatives d'adapter les principes structuralistes à l'étude de l'objet ${ }^{31}$, les théories portant sur la technique sont rares $^{32}$.

Les questions dépendent donc moins du matériel étudié que de l'actualité des domaines d'étude, or l'intérêt pour les objets varie au fil des années et tout sépare Vasari de Winckelmann, Wölfflin de Panofsky, Julius von Schlosser de Louis Marin. Cet article ne développe pas toutes les possibilités d'investigations sur les reliquaires ${ }^{33}$ mais on retiendra que ces objets soulèvent des problèmes susceptibles d'intéresser des historiens de l'art, même si la qualité de ces objets ne les prédispose pas à une étude sur l'évolution des styles. Ils offrent aussi un intérêt pour des anthropologues ou des historiens de la religion, surtout si l'on regarde leur fonctionnement cultuel plus que leur classification formelle.

Nous nous demandions pour qui inventorier. Si un chercheur ne retient pour son compte que ce qui l'intéresse ou des éléments qui ne seraient pas utilisables par d'autres, un service public ne peut se satisfaire d'une telle position.

Qu'inventorier alors, que consigner du culte des reliques lors d'un inventaire ? Il importe de prendre conscience de la corrélation entre les questions que l'on pose et les indications que l'on relève. Préparer le travail de l'ensemble des chercheurs relève de l'utopie parce qu'il est impossible d'anticiper les préoccupations de demain. Pour autant, faut-il renoncer à toute ambition scientifique ? L'inventaire peut offrir davantage qu'un relevé systématique de ce qui est susceptible d'intéresser l'érudition. Sans prétendre à recouvrir la totalité des champs de la recherche, il peut enregistrer ce que l'on estime être le plus profitable, ce qui suppose d'une part une connaissance préalable et précise de l'état des études savantes, de l'autre un choix, car il est impossible de tout noter.

Un tel parti n'est pas sans conséquences. Conséquences sur l'organisation, puisqu'il suggère une centralisation des questions scientifiques, nécessaire à un traitement sériel de l'information et à une prospection étendue dans plusieurs régions, ainsi qu'un travail concomitant en archives. Conséquences aussi sur le travail quotidien, notamment parce qu'il oblige à reprendre régulièrement les opérations d'inventaire, au fur et à mesure que se déplacent les centres d'intérêt.

59 Comment inventorier les reliquaires ? D'un cas a priori très concret l'on en vient à poser la question de la nature de la recherche scientifique, et de sa rentabilité. Celle-ci n'est pas financière, et inventaire patrimonial et scientifique ne présentent que peu de points communs.

60 Mais parce que la recherche n'est pas figée, parce qu'elle ne se contente pas de relevés définis une fois pour toutes, elle renouvelle en permanence les questions sur l'être humain, ses créations, ses croyances, son mode de vie, et cette connaissance du passé seule livre les clefs du monde dans lequel nous évoluons. C'est précisément cette capacité à s'interroger sur la place de l'homme, à proposer des modèles existentiels et sociétaux, qui fait de la recherche, donc des services d'inventaire, un véritable service public.

61 L'inventaire des églises et le dépouillement des périodiques ou des monographies d'édifices ont permis de relever 3400 reliques, présentes ou mentionnées, correspondant à environ 800 saints. Ces chiffres ne donnent qu'une idée partielle du nombre des reliques 
qui revinrent à Paris, dans la mesure où l'enquête a privilégié les églises paroissiales. Un peu plus de 840 contenants ont été recensés lors de cette enquête.

\section{ANNEXES}

Liste des édifices dont l'inventaire serait à faire ou à reprendre

Si la plupart des églises paroissiales appartenant à la Ville de Paris ont fait l'objet d'un inventaire approfondi, d'autres lieux n'ont pas été visités, ou d'une manière que l'auteur estime insuffisante, le plus souvent en raison des réticences de leurs responsables. Le nombre d'objets collecté est au demeurant tel, qu'il est statistiquement improbable qu'un complément d'inventaire puisse fortement modifier les conclusions.

Cœur Immaculé de Marie, 51 bis, rue de la Pompe, $\mathrm{XVI}^{\mathrm{e}}$

Marie-Médiatrice, 48 bis, boulevard Sérurier, XIX

Médaille Miraculeuse, 140 rue du Bac, $\mathrm{VII}^{\mathrm{e}}$

Missions étrangères (hors salle exposition), 128 , rue du Bac, $\mathrm{VII}^{\mathrm{e}}$

Notre-Dame, 6, place du Parvis Notre-Dame, IV

Notre-Dame-de-l'Assomption, place Maurice-Barrès, $\mathrm{I}^{\mathrm{er}}$

Notre-Dame-Auxiliatrice, 15, rue du Retrait, $\mathrm{XX}^{\mathrm{e}}$

Notre-Dame-du-Bon-Conseil, 140, rue de Clignancourt, XVIII ${ }^{\mathrm{e}}$

Notre-Dame-de-la Croix, 4, rue d'Eupatoria, $\mathrm{XX}^{\mathrm{e}}$

Notre-Dame-d'Espérance, 31, rue Dombasle, XV

Notre-Dame-de-Grâce-de-Passy, 10, rue de l'Annonciation, XVI

Notre-Dame-de-Lourdes, 130, rue Pelleport, $\mathrm{XX}^{\mathrm{e}}$

Notre-Dame-du-Perpétuel-Secours, 55, boulevard de Ménilmontant, $\mathrm{XX}^{\mathrm{e}}$

Notre-Dame-Réconciliatrice-de-la-Salette, 55, boulevard de Belleville, $\mathrm{XI}^{\mathrm{e}}$

Notre-Dame-Réconciliatrice-de-la-Salette, 57, boulevard de Belleville, $\mathrm{XI}^{\mathrm{e}}$

Notre-Dame-du-Saint-Sacrement, 20, rue Cortambert, $\mathrm{XVI}^{\mathrm{e}}$

Notre-Dame-de-la-Salette, 38, rue de Cronstadt, XV

Notre-Dame-du-Val-de-Grâce, 1, rue Alphonse-Laveran, $\mathrm{V}^{\mathrm{e}}$

Orphelinat Saint-Charles, 310, rue de Vaugirard, $\mathrm{XV}^{\mathrm{e}}$

Saint-Antoine-des-Quinze-Vingts, 66, avenue Ledru-Rollin, XII ${ }^{\mathrm{e}}$

Saint-Augustin, place Saint-Augustin, VIII ${ }^{e}$

Saint-Charles-de-la-Croix-Saint-Simon, 16, rue de la Croix-Saint-Simon, XX 
Saint-Éloi, 3, place Maurice de Fontenay, XII ${ }^{\mathrm{e}}$

Saint-Esprit, 1, rue Cannebière, XII ${ }^{\mathrm{e}}$

Saint-Étienne-du-Mont, place Sainte-Geneviève, $\mathrm{V}^{\mathrm{e}}$

Saint-Germain-des-Prés, 1, place Saint-Germain-des-Prés, VI ${ }^{\mathrm{e}}$

Saint-Ignace, 33, rue de Sèvres, VI $^{\mathrm{e}}$

Saint-Jacques, 20, rue des Tanneries, XIII ${ }^{\mathrm{e}}$

Saint-Jean-de-Montmartre, 19, rue des Abbesses, XVIII ${ }^{\mathrm{e}}$

Saint-Jean-Baptiste-de-Belleville, 139, rue de Belleville, XIX ${ }^{\mathrm{e}}$

Saint-Joseph-Artisan, 214, rue La Fayette, $\mathrm{X}^{\mathrm{e}}$

Saint-Joseph-des-Carmes, 70, rue de Vaugirard, VI $^{\mathrm{e}}$

Saint-Leu-Saint-Gilles, 92 bis, rue Saint-Denis, $\mathrm{I}^{\mathrm{er}}$

Saint-Louis, 123, rue de la Grange-aux-Belles, $\mathrm{X}^{\mathrm{e}}$

Saint-Louis de l'École Militaire, 13, place Joffre, VII ${ }^{\mathrm{e}}$

Saint-Louis-des-Invalides, Hôtel des Invalides, VII ${ }^{\mathrm{e}}$

Saint-Martin-des-Champs, 36, rue Albert-Thomas, $\mathrm{X}^{\mathrm{e}}$

Saint-Pierre-de-Montrouge, 82, avenue du Général-Leclerc, XIV

Sainte-Bernadette, 12, avenue de la Porte-de-Vincennes, XII

Sainte-Bernadette, 4, rue d'Auteuil, $\mathrm{XVI}^{\mathrm{e}}$

Sainte-Claire, 179, boulevard Sérurier, XIX ${ }^{\mathrm{e}}$

Sainte-Famille, 46, rue de Montreuil, $\mathrm{XI}^{\mathrm{e}}$

Sainte-Geneviève, 18 , rue Claude-Lorrain, $\mathrm{XVI}^{\mathrm{e}}$

Sainte-Hélène, 102, rue du Ruisseau, XVIII ${ }^{\mathrm{e}}$

Sainte-Marie, abbaye, 3, rue de la Source, $\mathrm{XVI}^{\mathrm{e}}$

Sainte-Thérèse, 71 bis, rue Boissière, $\mathrm{XVI}^{\mathrm{e}}$

Sainte-Trinité, 3, rue de la Trinité, IX ${ }^{\mathrm{e}}$

$\underline{\text { Archives dépouillées }}$

Conservation des œuvres d'art religieuses et civiles de la Ville de Paris (COARC) : 55, rue des Francs-Bourgeois, 75004 Paris.

Archives de l'Archevêché de Paris : 4, rue de l'Asile Popincourt, 75011 Paris.

Archives nationales (quelques séries seulement).

Archives des Capucins de Paris : 26 rue Boissonade, 75014.

Archives de l'église Saint-Sulpice, place Saint-Sulpice, 75006.

Archives de l'église Saint-Eustache, place du Jour, 75001.

Bibliothèque du Saulchoir, 43 rue de la Glacière, 75013. 
Archives des Filles de la Charité, 140 rue du Bac 75007 : accès difficile et très limité à un fonds qui semble extrêmement riche.

Archives de la Congrégation de la Mission, 95 rue de Sèvres, 75006.

Archives jésuites, 15, rue Raymond Marcheron, Vanves, 92170.

Services des archives de la Maison des Lazaristes (Congrégation des Missions), 95 rue de Sèvres, 75006.

Fonds des archives de l'orfèvre Lesage, musée Carnavalet, 23, rue de Sévigné, 75003 Paris.

\section{NOTES}

1. - Cet article s'appuie sur GAGNEUX, Yves. Reliques et reliquaires à Paris. (XIX ${ }^{e}-X X^{e}$ siècle). Paris : Éd. du Cerf, 2007, (coll. «Histoire religieuse de la France»), où l'on trouvera le détail des différentes références.

2. - 55 rue des Francs-Bourgeois, 75004 Paris.

3. - Ce n'est bien évidemment pas le cas et les campagnes photographiques s'accompagnent d'un important travail documentaire, l'ensemble étant reporté sur une base de données.

4. - Elles se limitent à ce qui permet de faciliter le repérage, tant pour la gestion quotidienne qu'en cas de disparition.

5. - Des éléments historiques ou politiques s'y grefferont éventuellement.

6. - GAGNEUX, Yves. Reliques et reliquaires à Paris. (XIX ${ }^{e}-X X^{e}$ siècle). Paris : Éd. du Cerf, 2007, (coll. " Histoire religieuse de la France »). Le corpus comprend plus de 3400 reliques, correspondant à plus de 800 saints.

7. - En province, ce sont des églises entières que l'on construit pour abriter des reliques, ainsi l'église Sainte-Clotilde à Reims édifiée à l'occasion du centenaire du baptême de Clovis en 1896 pour devenir le « reliquaire de la France chrétienne ».

8. - Concile de Trente « Décret sur l'invocation, la vénération et les reliques des saints et sur les saintes images", (session XXV, 3 et 4 décembre 1563), extrait de Le Saint Concile de Trente œcuménique et général célébré sous Paul II Jules II et Pie IV souverains pontifes, nouvellement traduit par M. l'abbé Chanut, $3^{\mathrm{e}}$ édition, Paris, Sébastien Mabre-Cramoisy, 1686.

9. - S.C. Rit., 1234 ad $2^{\circ}$.

10. - Can. 1281,82 ; S.C. Rit. 1333 ad $14 ; 1334$ ad 3.

11. - On peut proposer une définition anthropologique de la relique : les restes de la personne élue ou de ses biens, dans la mesure où ils servent à établir des relations, ce qui étend ce concept aux souvenirs laïcs. Voir GAGNEUX, Yves. Reliques et reliquaires à Paris. (XIX ${ }^{e}-X X^{e}$ siècle). Paris : Éd. du Cerf, 2007, (coll. « Histoire religieuse de la France »), p. 28.

12. - La législation canonique ou civile interdit l'appropriation des restes humains.

13. - « Reliquaire : Boite ou coffret précieux renfermant les reliques. »; « Châsse : Coffre où l'on garde les reliques d'un saint. » Le Petit Robert. Paris : S.E.P.R.E.T., 1975.

14. - Les reliques sont indispensables pour les autels mais une fois achevées les cérémonies de consécration, il ne leur est plus rendu de culte. On notera le cas particulier des autels dont le tombeau renferme effectivement des reliques, souvent placées derrière une vitre (corps saint présenté dans une figure de cire, ou petite châsse en métal).

15. - Par exemple dans la base Palissy pour l'Inventaire général du patrimoine culturel. (http:// www.culture.gouv.fr/culture/inventai/patrimoine/). On peut aussi consulter des études d'ensemble, par exemple pour Longpont-sur-Orge: RÉALE, Michel. La Basilique de Longpont. Étampes : Le Soleil Natal, 1988 ; CHABIN, Marie-Anne. Le Reliquaire de la basilique Notre-Dame-deBonne-Garde de Longpont-sur-Orge (canton de Montlhéry-Essonne) $19^{e}$ siècle, présentation et inventaire. 
Étude dactylographiée réalisée par le conservateur des antiquités et objets d'art de l'Essonne, 1988.

16. - Selon un symbolisme élémentaire pour les gens d'Église, le rouge est parfois associé au martyre, le blanc ou le doré à la pureté, le bleu à Marie. Sur un objet donné, le rapport reste toujours incertain mais un ample corpus permet de dégager des tendances.

17. - Il est parfois nécessaire d'enlever quelques vis pour parvenir au sceau.

18. - Voir par exemple TAUZIN, Henri. Armorial des cardinaux archevêques et évêques contemporains de France avec 86 écussons gravés. Paris, 1874 ; 1886; $1887 ; 1888$; Saint-Saud. Blasons, sceaux et devises des archevêques et évêques français de 1906. Vannes, 1906 ; COSSON, André. Armorial des cardinaux, archevêques et évêques français actuels résidentiels et titulaires au 1er janvier 1917. Paris, 1917.

19. - Les authentiques précisent le nom du dignitaire, la date de la reconnaissance et donnent parfois des indications sommaires sur la manière dont la relique est présentée.

20. - VAN GENNEP, Arnold. Culte populaire des saints en Savoie. Paris : G.-P. Maisonneuve et Larose, 1973 (recueil d'articles publiés de 1916 à 1939).

21. - On notera que les inscriptions portées sur le reliquaire peuvent donner une indication chronologique, en particulier pour les saints dont on connaît les dates de béatification et de canonisation : les mentions «bienheureux » ou "saint » donneront un intervalle, une date post quem ou ante quem.

22. - Les authentiques peuvent en effet avoir été déposées dans les archives diocésaines.

23. - On connaît le récit de l'apparition de Jésus à Marguerite-Marie en 1675, lui montrant son cœur en disant « Voici ce cœur qui a tant aimé les hommes... ».

24. - Monument de Jean-Marie Vianney, église d'Ars-sur-Formans.

25. - Voir, sous la direction de BOYER, Raymond et GRÉVIN, Gilles. Une sainte provençale du XIV siècle : Roseline de Villeneuve : enquête sur sa «momie ». Paris : de Boccard, 2002.

26. - Pour saint Remi, saint Martin et saint Jean-Marie Vianney.

27. - Le premier pape et la patronne de la France, les emblèmes de l'Église catholique française, indépendante de Rome, fondée en janvier 1831 par l'abbé François-Ferdinand Chatel. L'église Sainte-Rita se trouve 27, rue François Bonvin, 75015 Paris.

28. - Catalogues commerciaux de mobilier ou d'objets religieux du $19^{e}$ siècle, liste établie par Frédéric Bertrand de Puyraimond, Fabienne Gilbert, Nathalie Malbet et Anne Raffin, en collaboration avec Nicole Blondel.

29. - MUEL, Francis, DESVIGNES-MALLET, Chantal, PALOUZIÉ, Hélène, RÉVEILLON, Élisabeth, VERGNE, Sophie et HAMELIN, Liliane. De la petite cuillère... au reliquaire ou l'inventaire de l'orfèvrerie, In Situ n6, septembre 2005.

30. - GAGNEUX, Yves. «Entre culte et histoire, le reliquaire du tombeau de sainte Geneviève à Saint-Étienne-du-Mont ». Corps saints et lieux sacrés de l'Europe moderne, ouvrage collectif dirigé par Philippe Boutry, Pierre-Antoine Fabre et Dominique Julia, EHESS, 2009.

31. - Par exemple l'ensemble des travaux d'André Leroi-Gourhan qui développent les positions plus générales exprimées entre autres par SIMONDON, Gilbert. Du mode d'existence des objets techniques. Paris : Aubier, 1958 ou LEVI-STRAUSS,Claude. Anthropologie structurale. Paris: Plon, 1958.

32. - Une théorie générale tendant à proposer une refondation des sciences humaines accorde une part considérable à la technique. Voir GAGNEPAIN, Jean. Du Vouloir-Dire, traité d'épistémologie des sciences humaines, I Du signe. De l'outil. Paris : Livre et communication, 1990 [1 1 ère édition 1981]; Du vouloir dire. III, Guérir l'homme, former l'homme, sauver l'homme: traité d'épistémologie des sciences humaines. Bruxelles: De Boeck université, 1995. Les conséquences sur l'histoire de l'art et l'archéologie ont été développées par BRUNEAU, Philippe et BALUT, Pierre-Yves. Artistique et archéologie, Mémoires d'Archéologie générale, 1-2. Paris : P.U.P.S., 1997. 
33. - Voir GAGNEUX, Yves. Reliques et reliquaires à Paris. (XIX ${ }^{e}-X X^{e}$ siècle). Paris : Éd. du Cerf, 2007, (coll. "Histoire religieuse de la France»), avec une présentation plus étendue. Les exemples parisiens ne se prêtent néanmoins pas à toutes les recherches qu'autorisent les bases théoriques retenues dans cet ouvrage.

\section{RÉSUMÉS}

Les tentatives d'inventaire scientifique des reliquaires des deux derniers siècles conservés dans les églises parisiennes mettent en évidence l'inadaptation des définitions traditionnelles et conduisent à renoncer à définir ce qu'est un reliquaire, au profit d'une étude systématique des éléments liés au culte des reliques. Cette étude passe classiquement par le relevé des inscriptions et des représentations, mais conduit également à s'intéresser aux identités sociales qui se manifestent autour des aménagements autour des reliques, qu'il s'agisse d'individualités, de caractéristiques de groupes, voire de concepts. Elle impose aussi de relever ce qui organise matériellement le culte, et ses usages, intégrant le relevé des témoignages écrits, graphiques ou photographiques. Un inventaire scientifique doit répondre à des questions qui évoluent et devra donc sans cesse être repris. L'ampleur des tâches oblige à choisir ses objectifs, ce qui exige une connaissance précise de la recherche à son plus haut niveau.

\section{INDEX}

Mots-clés : inventaire patrimonial, inventaire scientifique, Paris, relique, reliquaire, sainteté, canonisation, béatification, reliques insignes, reliques corporelles, reliques réelles, reliques représentatives, chasse, sceau, authentique, corps saint, catacombes, Talrich, histoire, culte, cérémonie, décor éphémère, ex-voto, néo-gothique, analyse, théorie

\section{AUTEUR}

\section{YVES GAGNEUX}

directeur de la Maison de Balzac yves.gagneux@paris.fr 